Article

\title{
Frequency Resolved Partial Discharges Based on Spectral Pulse Counting
}

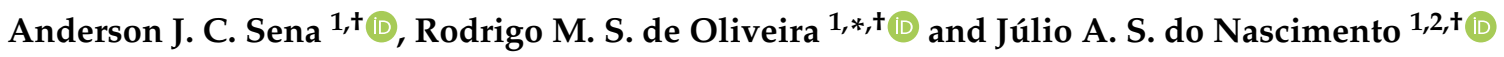 \\ 1 Electrical Engineering Graduate Program (PPGEE), Federal University of Pará, Belém 66075-110, Brazil; \\ ajcsena@gmail.com (A.C.J.S.); salhebjulio@gmail.com (J.A.S.d.N.) \\ 2 Eletrobras Eletronorte, Rodovia Arthur Bernardes 2175, Belém 66115-000, Brazil \\ * Correspondence: rmso@ufpa.br \\ + These authors contributed equally to this work.
}

check for updates

Citation: Sena, A.J.C.; de Oliveira, R.M.S.; do Nascimento, J.A.S Frequency Resolved Partial Discharges Based on Spectral Pulse Counting. Energies 2021, 14, 6864. https://doi.org/10.3390/en14216864

Academic Editor: Zhijin Zhang and Hualong Zheng

Received: 5 September 2021

Accepted: 24 September 2021

Published: 20 October 2021

Publisher's Note: MDPI stays neutral with regard to jurisdictional claims in published maps and institutional affiliations.

Copyright: (c) 2021 by the authors. Licensee MDPI, Basel, Switzerland. This article is an open access article distributed under the terms and conditions of the Creative Commons Attribution (CC BY) license (https:// creativecommons.org/licenses/by/ $4.0 /)$.

\begin{abstract}
A partial discharge (PD) classification methodology based counting PD pulses in the spectral domain is proposed and presented in this paper. The spectral counting data are processed using the proposed PD Spectral Pulse Counting Mapping technique (PD-SPCM), which leads to a Frequency-Resolved Partial Discharges (FRPD) map. The proposed map is then used for PD detection and classification. In this work, corona and slot FRPDs are presented in frequency bands up to $500 \mathrm{MHz}$, obtained from laboratory measurements performed using two hydro-generator stator bars. The electromagnetic signals from the PDs were captured using a patch antenna designed for this purpose and a spectral analyzer. The corona and slot PDs were chosen because one can be mistakenly classified as the other because they may present similar Phase Resolved PD (PRPD) maps and may occupy shared spectral bands. Furthermore, corona and slot PDs can occur concurrently. The obtained results show that the corona and slot PDs can be properly identified using the developed methodology, even when they occur simultaneously. This is possible because, as it is experimentally demonstrated, corona and slot PDs have appreciable levels of spectral pulse counting in particular bands of the frequency spectrum.
\end{abstract}

Keywords: hydro-generators; partial discharges; PD Spectral Pulse Counting Map (PD-SPCM); microstrip antenna; UHF method; Frequency Resolved Partial Discharges (FRPD)

\section{Introduction}

Rises in amplitude and occurrence rates of partial discharges (PDs) in insulation dielectrics of hydro-generators stator bars indicate electrical problems that can compromise the operational safety of this kind of high-voltage equipment [1-3]. If the evolution of PD is not investigated, generation outages may take place, along with the consequent huge losses for society, which depends on electric power.

High sinusoidal voltage is produced by a generator, which induces a high electric field in the rotating machine and surroundings, including the air, the electrical insulation materials and surfaces of the generator structure. Therefore, PDs can be associated with several problems, including dielectric rupture of gases confined in stator bar insulation internal voids, the degradation of the semiconductive layer due, for instance, to mechanical or electrical stresses, and the presence of undesired objects in the stator winding [2]. Discharge activities evolve gradually over the years, which can be identified and quantified by measuring acoustic emissions [4,5], electrical PD signals [6,7], and electromagnetic radiation [8]. If the electrical problems PDs indicate are not monitored and treated, they can evolve to reach a state of complete unavailability of the hydro-generator [2,9-11].

In general, several features have been used to extract information from PD signals, such as Weibull probability function [12], cross-wavelet transforms [13], rough sets [14], time-of-pulse travel between sensors, and time-frequency maps [15]. A feature frequently used to identify PD sources from hydro-generators is the PRPD (Phase-Resolved Partial 
Discharge) [16,17], which contains PD peaks distributed over the generator $50 \mathrm{~Hz}$ or $60 \mathrm{~Hz}$ power signal phase, such that the peaks are accumulated over time (at least during $30 \mathrm{~s})$. The patterns seen in PRPD maps are standardized in [6] for each type of hydrogenerator PD.

In certain circumstances, however, PRPDs may provide limited classification possibilities [7]. For instance, if slot and corona PDs occur simultaneously, their PRPD clouds will be intertwined or superimposed because the phases they produce are coincident $[6,18]$. Thus, their PRPDs would overlap, making it difficult to properly identify them only by inspecting the PRPD cloud; i.e., it would be tricky or virtually impossible to determine the presence of both faults or one of them. Furthermore, although specific PD sources are manifested in specific frequency ranges [19], when two faults are simultaneously occurring, their frequency bands may intersect such that one is contained within the spectrum of the other. This is the case of simultaneous slot and corona PDs, in which it is also not possible to distinguish the PD sources just by inspecting the resulting spectrum (or PRPD).

In this paper, a new methodology is proposed in which spectral PD count is introduced, producing a novel tool for statistical analysis of PDs-the Partial Discharge Spectral Pulse Counting Mapping technique (PD-SPCM) — which leads to a Frequency Resolved Partial Discharges (FRPD) map. The proposed method is based on spectral counting of PD pulses, with which one is able to identify the types of simultaneous faults producing the PDs, even if their spectra are in the same frequency bands and their PRPD clouds completely overlap each other. FRPD has been validated experimentally in this work.

\section{Slot and Corona Discharges}

Slot discharges occur when the semiconductive coating of the bar has high resistance or when friction on the coating, due to machine vibrations and wedge looseness, leaves the high-voltage bare insulation, causing a metallic grounded core [19]. Because of the bare metallic surface of the lamination, the initial availability of free electrons is sufficient to start an electron avalanche in the air gap, regardless of the voltage polarity. After some time, intense slot discharges, along with ozone and nitric acid produced as byproducts, starts to attack both the insulation and the core, causing oxidation. This can affect the availability of free electrons. Thus, the typical asymmetry on the slot PRPD map [6] becomes more pronounced a few hours after the initial slot PDs [2,19].

Corona discharges occur due air ionization produced by high electric fields, originating from the bar surface, usually at the junction between the semi-conductive material (used in the slot) and the stress-grading material (used in the winding output terminals) [19]. This type of activity causes degradation directly at the junction, leaving a deposit of white powder. This activity can evolve slowly over the years. In [19], it is shown experimentally that corona PRPD can change due to degradation of the junction or voltage increases.

In terms of spectral bands, it was determined experimentally in [19] that spectra of corona and slot PDs reaches up to $500 \mathrm{MHz}$. This means that, although slot PDs tend to produce higher amplitude levels [6], it may be difficult to distinguish slot discharges when they are occurring simultaneously with corona PDs simply by using the measured spectrum (as well as PRPDs [6]). PD energy was registered for frequencies over $1.5 \mathrm{GHz}$ in [20].

\section{Physics of Corona and Slot PDs}

Considering a stator bar of a hydro-generator subjected to the $60 \mathrm{~Hz}$ voltage produced, the higher the produced voltage, the greater the strength of the electric field in the semiconductive coating and at the junction among the slots and the winding output terminals, causing ionization of the surrounding air. This ionization occurs because, due the mentioned defects on the surfaces, the electric field rises to levels sufficiently high to extract electrons from the valence shells of gas atoms. Each removed electron is accelerated due to the action of the high electric field, causing collisions with the electrons of the other 
electrically neutral atoms of the gas, generating detachments of more electrons, and so on, leading to the so-called avalanche effect [21].

The phenomena just described produce a plasma, in which the ratio between the electron drift velocity $v_{d}$ and the electric field strength $E$ is represented as the electron mobility $\mu_{e}$ [22], which is thus given by

$$
\mu_{e}=v_{d} / E
$$

In addition, the plasma electrical conductivity $\sigma$ can be obtained by

$$
\sigma=e \cdot n_{e} \cdot \mu_{e}
$$

in which $n_{e}$ is the carrier concentration [22] and $e$ is the electron charge. Notice that, as is given by (1), a rise in $E$ leads to reduction of electronic mobility $\mu_{e}$ because the number of free electrons also increases.

In short, during the first stages of the ionization process, the conductivity given by (2) increases because the increase in carrier concentration $n_{e}$ is dominant over the reduction in $\mu_{e}$. Thus, while the electric field keeps rising, the number of free electrons capable of ionizing new atoms increases. During this initial ionization period, the current $i$, in the ionized region, will increase exponentially over time [22,23]. The voltage between the electrodes decreases because of the rapid increase in electrical conductivity of ionized air between the anode and the cathode. This behavior is consistent with what is predicted by Maxwell's equations; i.e., the electric field decreases as conductivity increases [24]. As a consequence, the ohmic resistance $R_{c}$ of the circuit shown in Figure 1 is subjected to increasing voltage due to the progressive increase in the current with $\sigma$ [22].

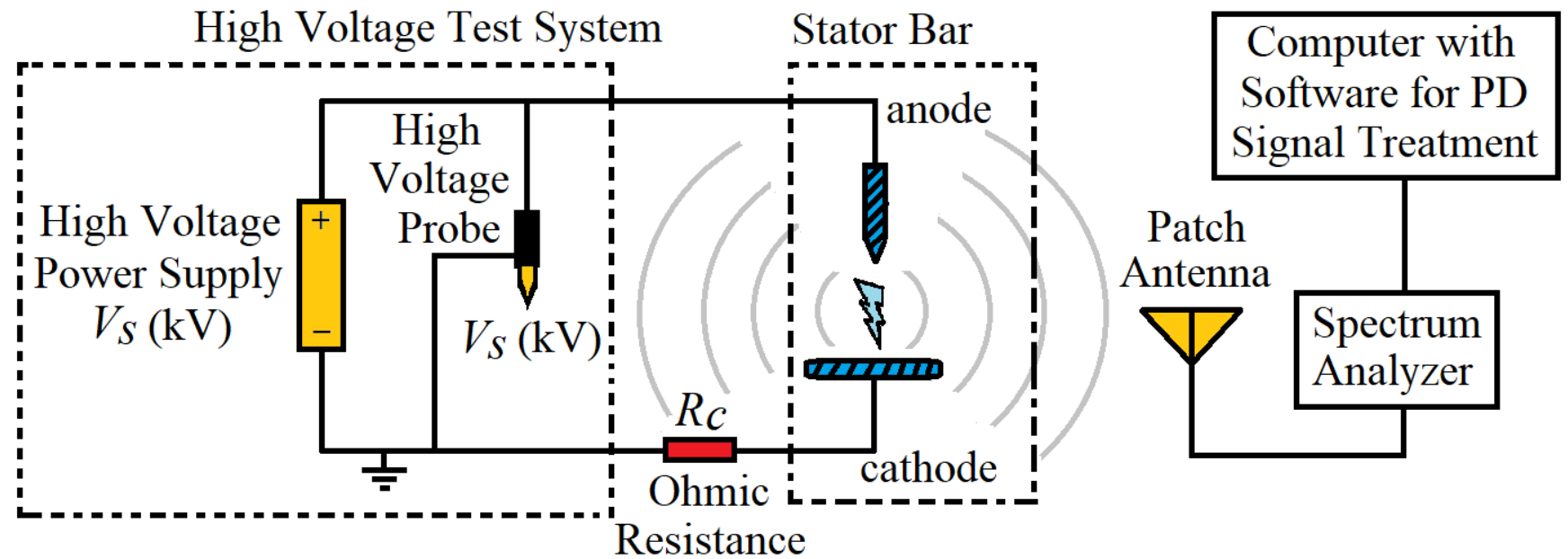

Figure 1. Representation of the discharge circuit proposed in [25]. The radiated electromagnetic field measurement setup (at right) is defined in this work.

Thus, the ionization process begins with intense electric field around the positive electrode, producing a distribution of positive ions around the anode and leading to a phenomenon called corona discharge (see Figure 2a). At this stage, the electric field intensity is high at the anode and decays to zero as one approaches the cathode (Figure 2b) [26]. As ionization continues due to the intense electric field, neutral atoms lose electrons that move towards the anode, causing the field to weaken in the vicinity of that electrode. This mechanism causes the field to increase around the regions farthest from the anode, around the tip of the just-ionized path, favoring the propagation of the ionization region towards the cathode (Figure 2c). As a result, the number of free electrons progressively increases due to the avalanche effect, leading to the consequent increase in current in the channel due to the increase in its conductivity [26]. The region around the ionized channel tip, called the streamer head, propagates towards the cathode as long as ions and electrons emerge 
more quickly than the weak electrical field of the conducting trail above the streamer head would be capable of. The conducting trail, which is referred to as streamer channel [26], electrically connects the streamer head to the anode.

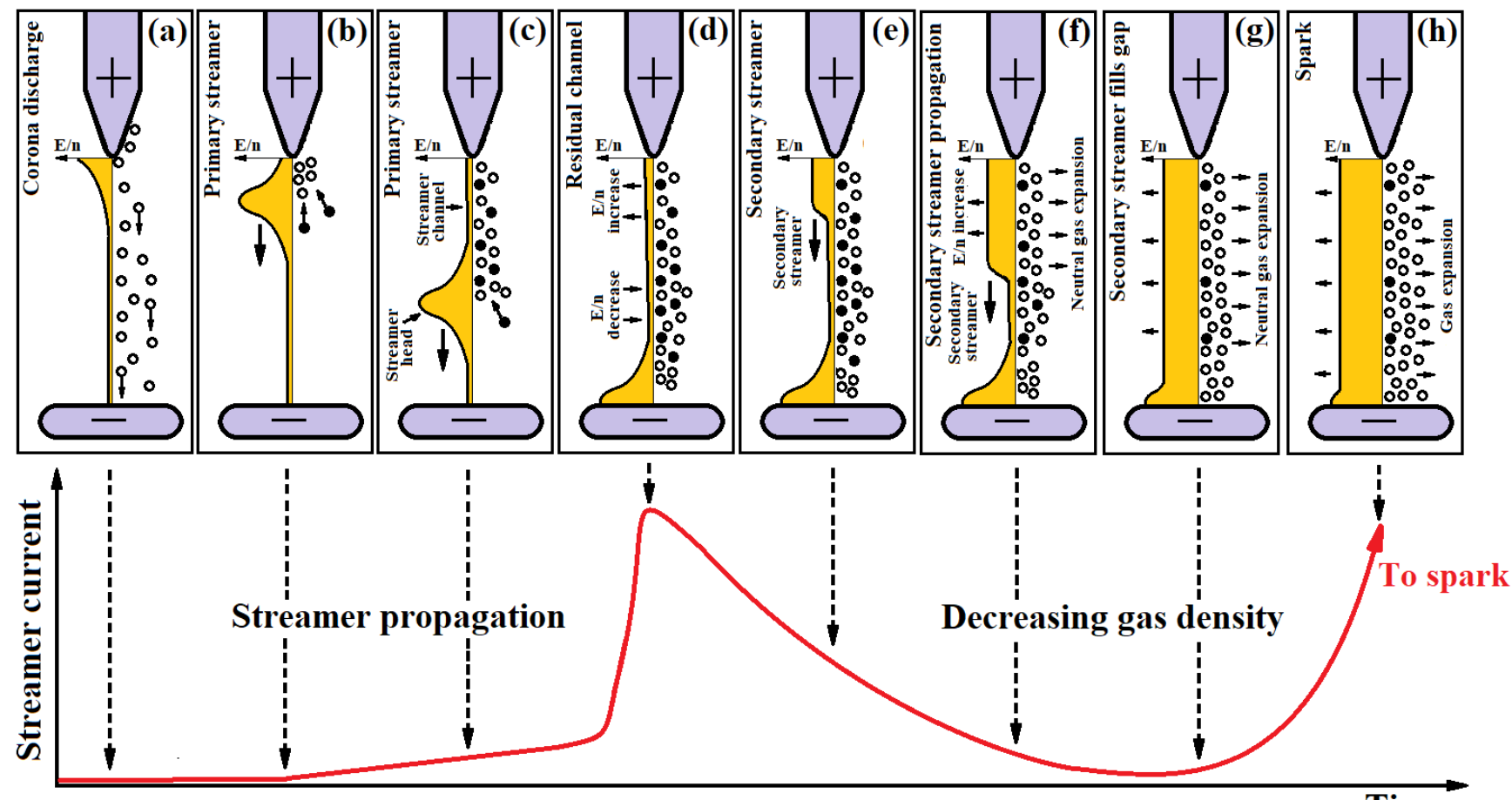

Time

Figure 2. Steps of primary and secondary streamers progressive developments [26]. The black circles represent electrons, while the white circles symbolize positively charged ions.

It should be noted that if the voltage supply level is not sufficient to maintain a high enough electric field in the gap between the electrodes, the streamer head is extinguished before reaching the cathode (as in corona dischargess). Otherwise, the streamer head moves quickly across the channel, closing the circuit between the electrodes (similarly to slot discharges [6]). As the streamer head reaches a distance of approximately one millimeter from the cathode (Figure 2d), a large number of electrons are emitted from the cathode due to intense ultraviolet radiation from the streamer head. This process leads to increases of thousands of volts of channel voltage during about one nanosecond, at least doubling the channel's average field during the process. The number of free electrons increases rapidly, leading to the emergence of a region with great ionization capability that propagates with high speed along the streamer channel towards the anode. This phenomenon favors equipotentialization along the residual streamer channel and produces the so-called return stroke, causing the current to reach its maximum value in this stage [26].

The electrical connection between the electrodes (Figure 2d), due to the ionized air filament, gives rise to a process leading to the so-called spark breakdown discharge [26]. This attachment instability precedes the formation of a secondary streamer, which consists of a progressive enlargement of the channel cross-section area due to a rise in temperature associated to the fast current increase produced by the high voltage from the source fully applied between the two ends of the channel. This process evolves into an equipotentialization state along the residual streamer channel (see Figure $2 \mathrm{e}-\mathrm{g}$ ). The secondary streamer is visually observed as a partial re-illumination of the streamer channel, usually starting at the anode at almost the same time that the streamer head makes contact with the cathode. The secondary streamer starts and moves in the channel created by the previous ionization processes, and although the increase in the channel cross-section area favors reducing $n_{e}$ and the electrical conductivity $\sigma$ as a consequence, the concurrent exponential decay of 
the current in the channel is also significantly dependent on the attachment of electrons to oxygen molecules [26].

When the excitation voltage is sufficient and fully applied across the ionized channel, the gas density $n$ is sufficiently reduced due to the expansion of the gas provided by the heating of the channel, leading to increase in $E / n$, thus allowing the propagation of the secondary streamer (Figure $2 \mathrm{f}$ ). The continuous gas expansion and the progressive $E / n$ increase allows the secondary streamer to cross the gap between the electrodes, forming a quasi-uniformly ionized domain (Figure $2 \mathrm{~g}$ ). Since $E / n$ reaches a certain magnitude, an abrupt new increase in the channel current towards a spark breakdown can be observed (see Figure 2h) [26].

\section{Discharges Due Sinusoidal Voltage Excitation}

In a hydroelectric generator, stator bars are often subjected to $50 \mathrm{~Hz}$ or $60 \mathrm{~Hz}$ sinusoidal electric fields. As illustrated in Figure 3, since voltage $V(t)$ is changing over time, stator discharges may or may not emerge [1], depending on present phase of the sinusoidal cycle. Discharge amplitude and duration may vary over the cycle with direct dependence on $V(t)$. Formation of corona and slot discharges are governed by the physical mechanisms previously described.

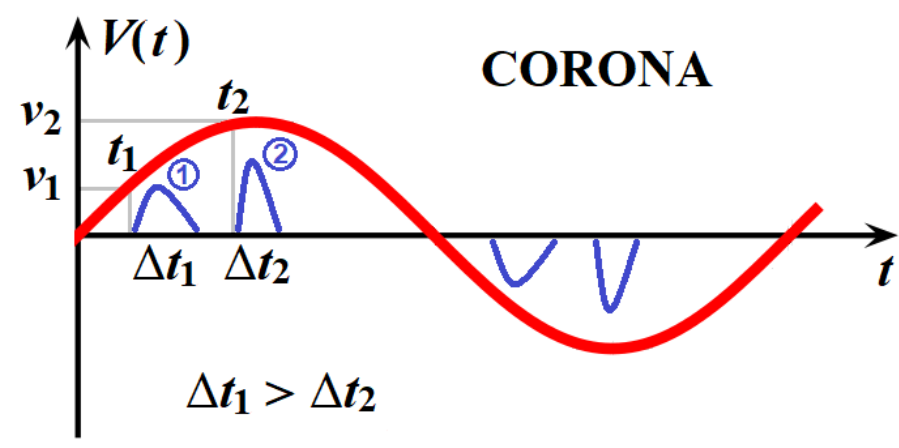

(a)

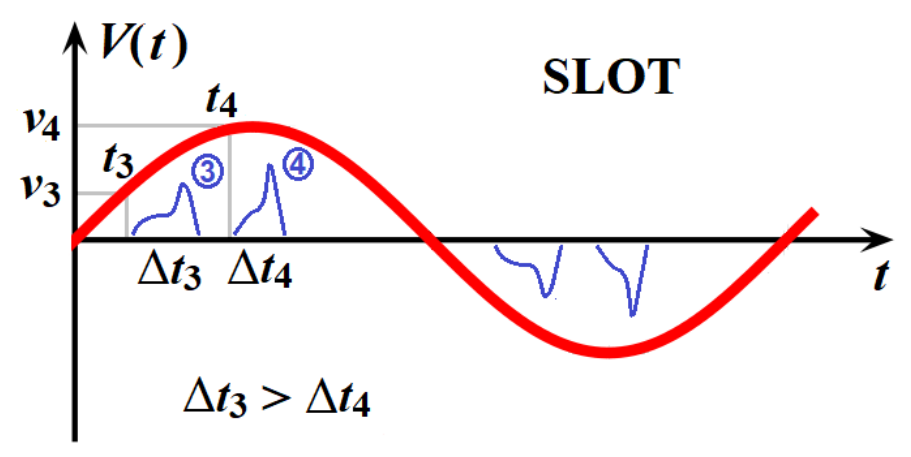

(b)

Figure 3. Ilustrations of partial discharges in a sinusoidal voltage signal: (a) corona and (b) slot.

As voltage magnitude increases, electric field also rises in the plasma channel and, as a consequence, the discharge current rise time is reduced before the spark stage takes place. Additionally, the streamer propagation speed increases [22,25-28]. Deionization takes place, as previously described. Then, with the increase in the generated voltage, the electric field rises again, and, since $E$ exceeds $E_{c}$, a new PD will occur at $t_{2}$, as shown in Figure 3a. Thus, during the evolution of the positive semicycle of the generated sinusoidal voltage, multiple PDs may occur with different amplitudes and time spams, i.e., the increase in the sinusoidal excitation voltage makes the partial discharge peaks occur in progressively shorter time 
intervals. As shown in Figure $3 a, \Delta t_{1}>\Delta t_{2}[22,25,27]$. In short, partial discharge pulses present increasing spectral bands and amplitudes as $V(t)$ increases in magnitude.

Notice that when corona discharges are developed, there is no electrical contact between electrodes due to the discharge channel because this type of discharge occurs between an energized electrode and air [6,29]. However, ionized arcs electrically connect electrodes during slot discharges [6]. For this reason, slot discharges are associated with higher discharge currents and broader frequency spectra because, once the streamer head reaches the cathode, as previously discussed, current rises more rapidly [25], i.e., steep slopes are seen in slot PD signals (occurring as the discharge channel electrically connects anode and cathode). This phenomenon increases frequencies composing the associated fields as a consequence. This is why slot PD functions have been depicted in Figure $3 \mathrm{~b}$ differently (with the steep slopes) from corona PDs seen in Figure 3a. These phenomena were experimentally observed in [25] using the discharge circuit illustrated in Figure 1 (excluding the antenna receiving setup).

Figure 4a shows the discharge currents obtained in [25] using 8.2, 7.2, and $6.2 \mathrm{kV}$ voltage excitation levels. Figure $4 \mathrm{~b}-\mathrm{d}$ shows wavelet transforms, calculated in this work, of PD current pulses in Figure 4a. Note that the higher the excitation voltage, the higher are the discharge current pulses and the shorter the time intervals necessary for current to reach its peak. Consequently, their spectral bands become larger, especially during the time periods of steep current slopes, as can be easily seen by comparing the wavelet transforms to each other and by observing their respective current signals evolving over time in Figure 4. Wavelet transforms $W\left(x(t), a, t^{\prime}\right)$ in Figure 4 were calculated by numerically evaluating $W\left(x(t), a, t^{\prime}\right)=\frac{1}{\sqrt{a}} \int_{-\infty}^{\infty} \psi\left(\frac{t-t^{\prime}}{a}\right) x(t) \mathrm{d} t$, in which $t$ is time, $a=1 / f, f$ is frequency, $t^{\prime}$ is the time shift factor, and $\psi(t)=\exp \left(-\frac{1}{2} t^{2}\right) \cos (2 \pi t)$ (the real-valued Morlet wavelet) [30].

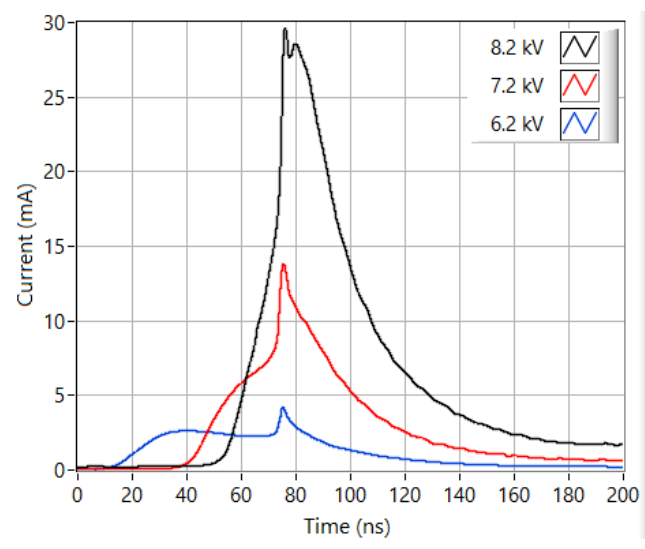

(a)

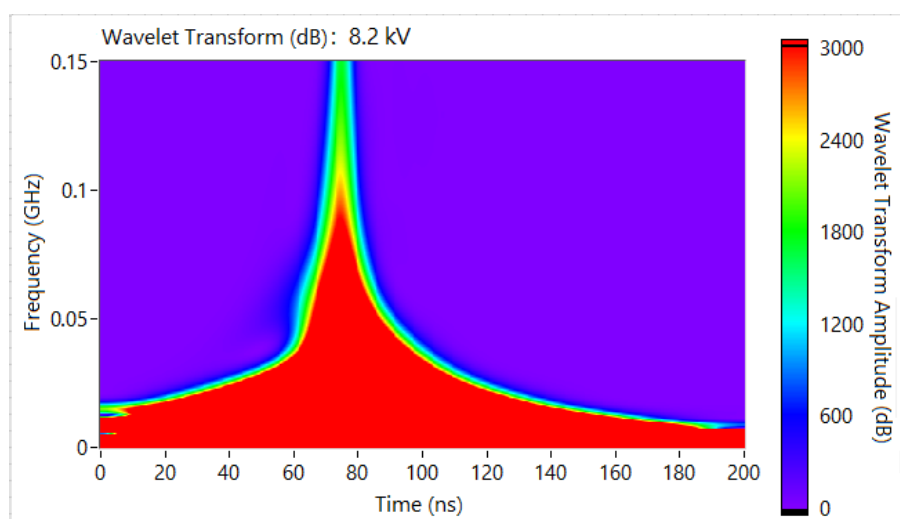

(b)

Figure 4. Cont. 


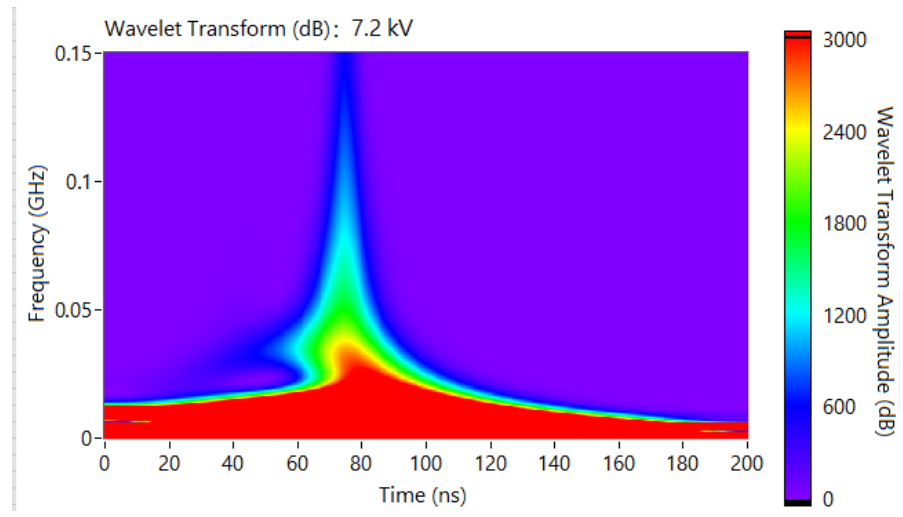

(c)

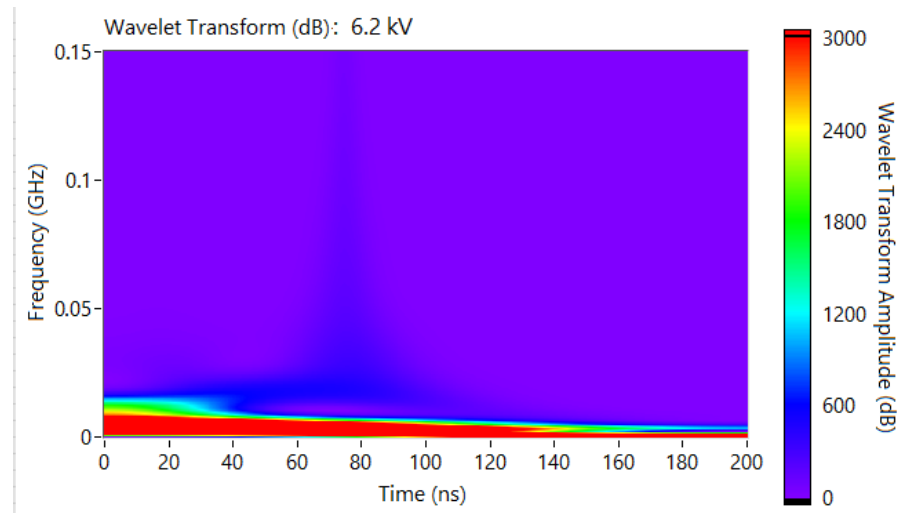

(d)

Figure 4. Discharge current pulses: (a) evolution of waveforms over time $(8.2 \mathrm{kV}, 7.2 \mathrm{kV}$ and $6.2 \mathrm{kV}$ pulses) [25], (b) wavelet transform of the $8.2 \mathrm{kV}$ pulse, (c) wavelet transform of the $7.2 \mathrm{kV}$ pulse, and (d) wavelet transform of the $6.2 \mathrm{kV}$ pulse.

\section{Frequency Resolved Partial Discharges (FRPD)}

In this section, basic concepts necessary for defining the proposed Partial Discharge Spectral Pulse Counting mapping technique, which leads to FRPD maps, are discussed and experimentally demonstrated. Furthermore, computer implementation of the proposed method is also examined.

\subsection{FRPD: Basic Concepts}

Since generated voltage sinusoidally oscillates in hydroelectric generators, amplitude and especially PD duration are not constant over the generator's $50 \mathrm{~Hz}$ or $60 \mathrm{~Hz}$ cycle. Because pulse duration directly affects spectral responses, PD count can be analyzed as a function of time and frequency, as shown by the experimental results obtained in this work, as shown in Figure 5. The spectrograms in Figure 5 show corona and slot PD pulses over the time-frequency domain, each registered within $8 \mathrm{~s}$ over a band defined between $20 \mathrm{MHz}$ and $160 \mathrm{MHz}$. The graphs show that the spectral PD pulses occur with different repetition levels when different frequencies are analyzed over time. This way, we have performed PD pulse count over time and frequency domains using data extracted from an spectrum analyzer employed to perform experimental measurements. The spectrum analyzer is connected to a patch antenna designed in this work for receiving high frequency $\mathrm{PD}$ radiated signals. The antenna design is detailed in Appendix A. 


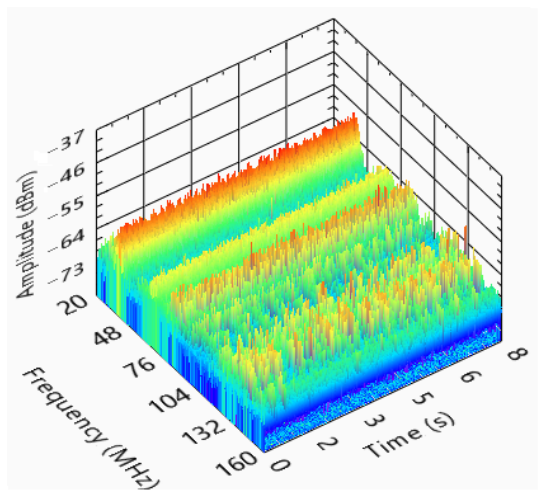

(a)

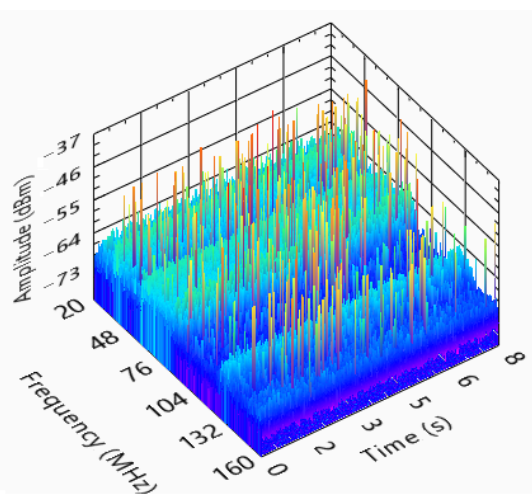

(b)

Figure 5. Spectrograms of (a) corona and (b) slot partial discharges. The spectrograms were registered in this work within eight seconds between $20 \mathrm{MHz}$ and $160 \mathrm{MHz}$.

Based on this experimental confirmation of the theoretical prediction, we developed in this work the Frequency Resolved Partial Dischage (FRPD) map (which is based on the developed Partial Discharge Spectral Pulse Counting technique), of which an illustrative example is shown in Figure 6. FRPD and its classification capabilities are the main contributions of this paper. In a short explanation, we may say that the map in Figure 6 is constructed by detecting pulses with amplitudes that are higher than a preestablished power threshold in the time-frequency domain (Figure 5) by counting the repeating pulses over time for each defined small registering frequency window and by averaging the amplitudes of the registered pulses in each registering frequency window. Normalized (percentage) spectral PD counting levels are represented by the colors of the dots in Figure 6.
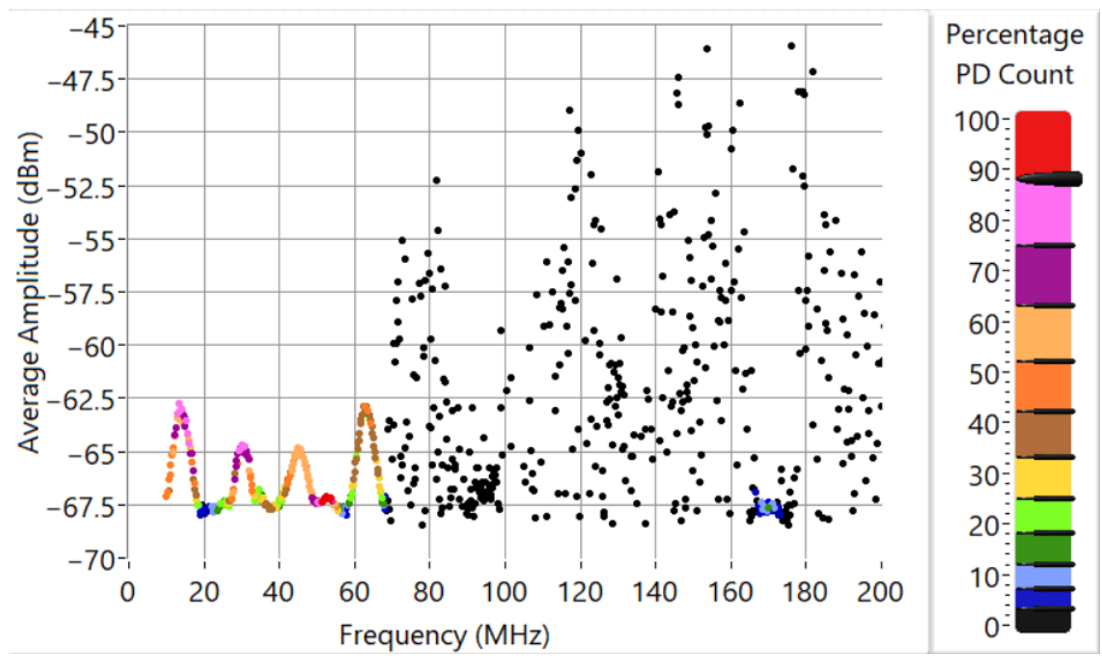

Figure 6. An illustrative example of the proposed FRPD map.

For the sake of clarification of the ideas just introduced, we show Figures 7 and 8 . Figure 7a shows that the pre-established noise threshold is set at $-60 \mathrm{dBm}$, which is considerably higher than the laboratory ambient power noise (noise can also be seen in Figure 7a). The graph in Figure 7b shows the first occurrence of a pulse at $387 \mathrm{MHz}$. During this occurrence, PD frequency and amplitude are registered, and the number of PD occurrences at $387 \mathrm{MHz}$ is increased (from zero to one in this case) and recorded. In a third time window in which the spectrum is measured, depicted by the graph in Figure 7c, the power noise threshold is once more exceeded at $387 \mathrm{MHz}$, and it is also crossed at $417 \mathrm{MHz}$ (for the first time). Then, PD frequency and amplitude recordings are made, and the occurrence count at $387 \mathrm{MHz}$ is once more incremented (from one to two). For 
$417 \mathrm{MHz}$, recordings of PD frequency and amplitude are also made, and the number of PD occurrences is set to one for that frequency (since, up to this time, a single PD occurence has been registered for $417 \mathrm{MHz}$ ). The PD frequency and amplitude recordings and spectral PD counting processes are repeated every time the signal amplitude is higher than the threshold for a given frequency (or frequencies). To obtain statistically consistent results, measurements need to be performed during a large number of sinusoidal cycles, i.e., over several seconds.

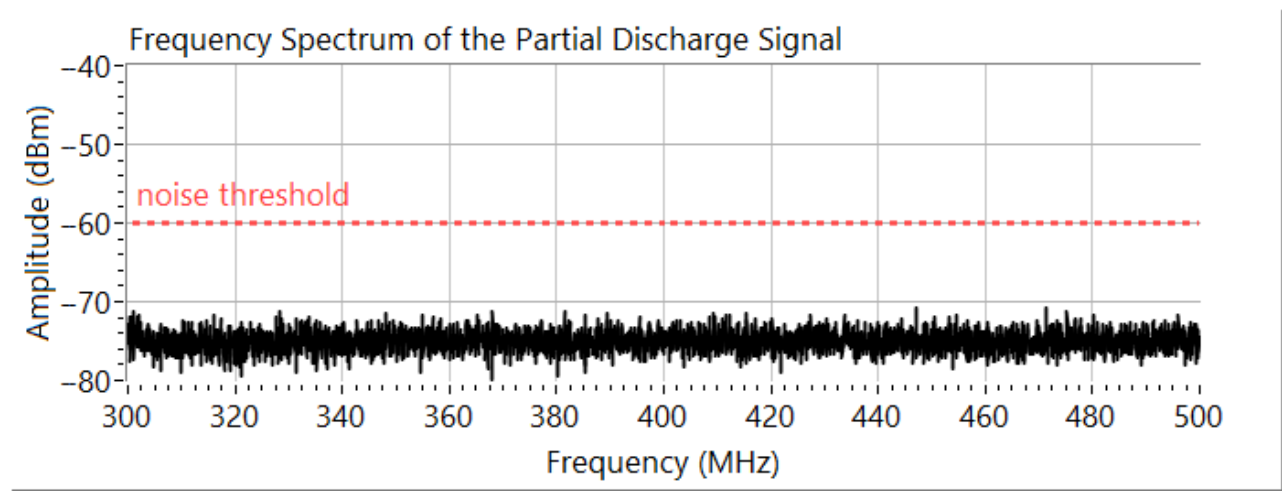

(a)

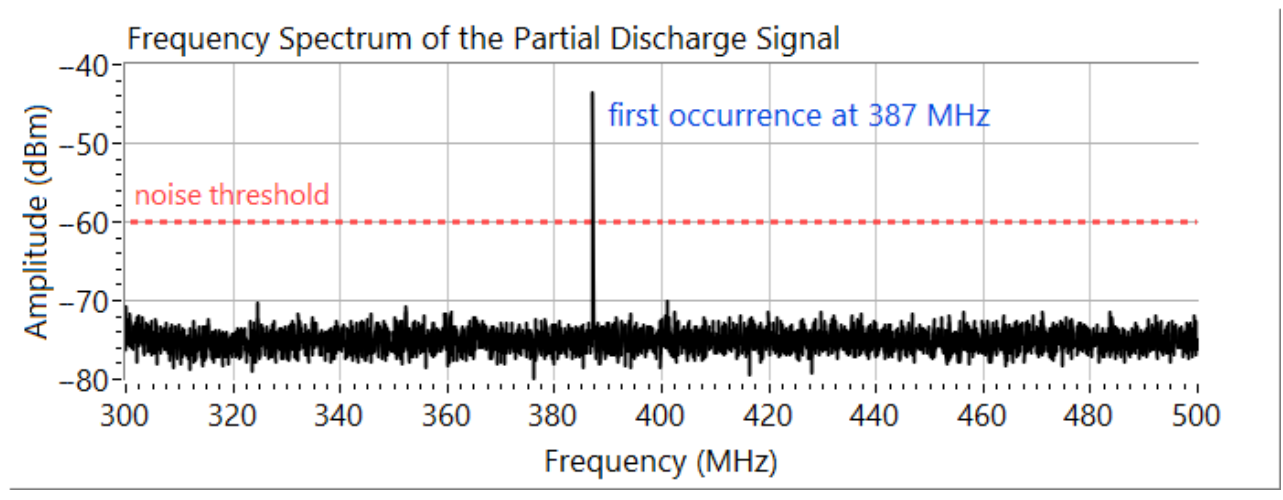

(b)

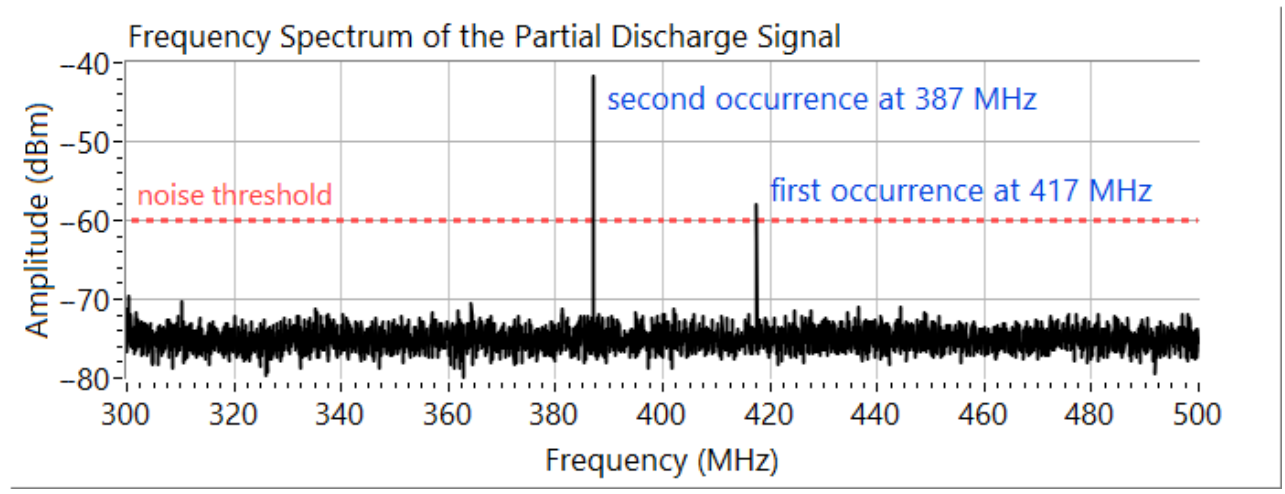

(c)

Figure 7. Spectral registering of PD pulses obtained at different times: (a) measurement with no occurrence records, at $t=t_{0} ;(\mathbf{b})$ detection of the first occurrence at $387 \mathrm{MHz}$, at $t=t_{1}$; and (c) detection of the second occurrence at $387 \mathrm{MHz}$ and of the first occurrence at $417 \mathrm{MHz}$, at $t=t_{2}$. 

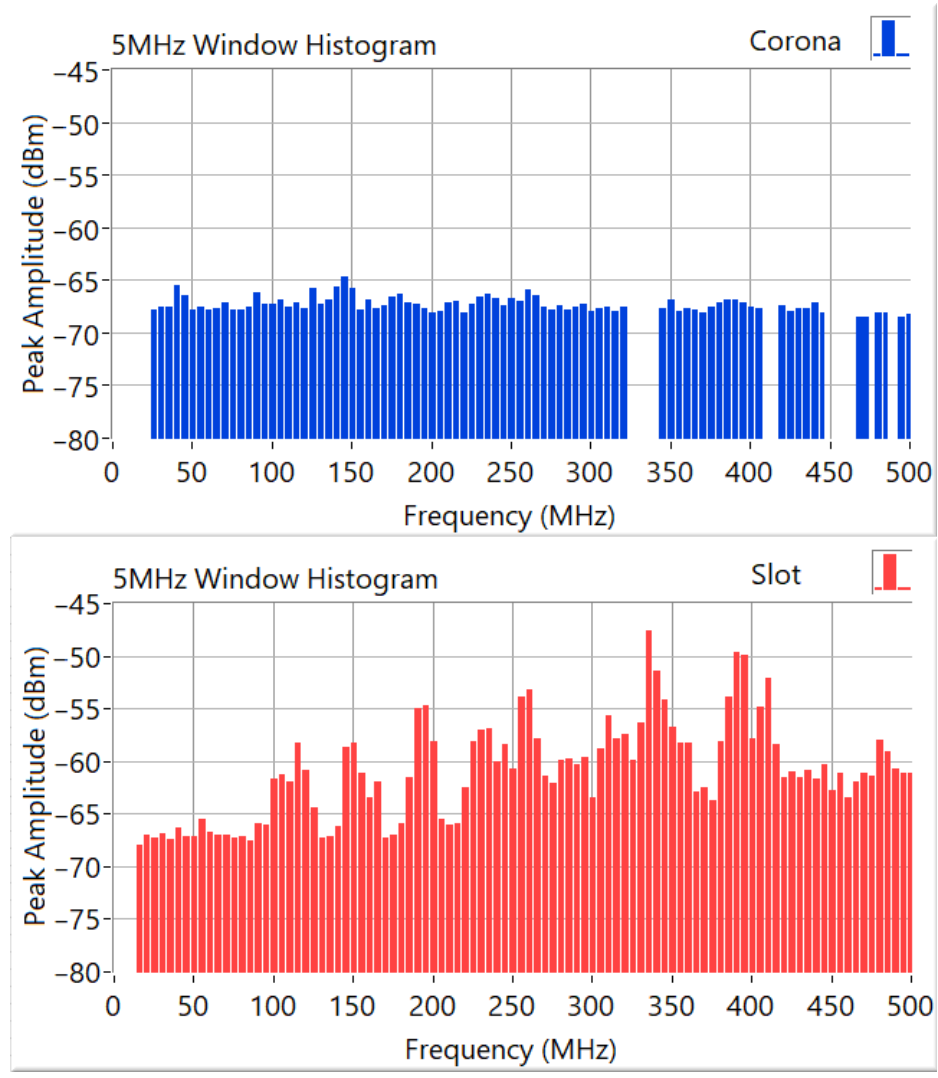

(a)
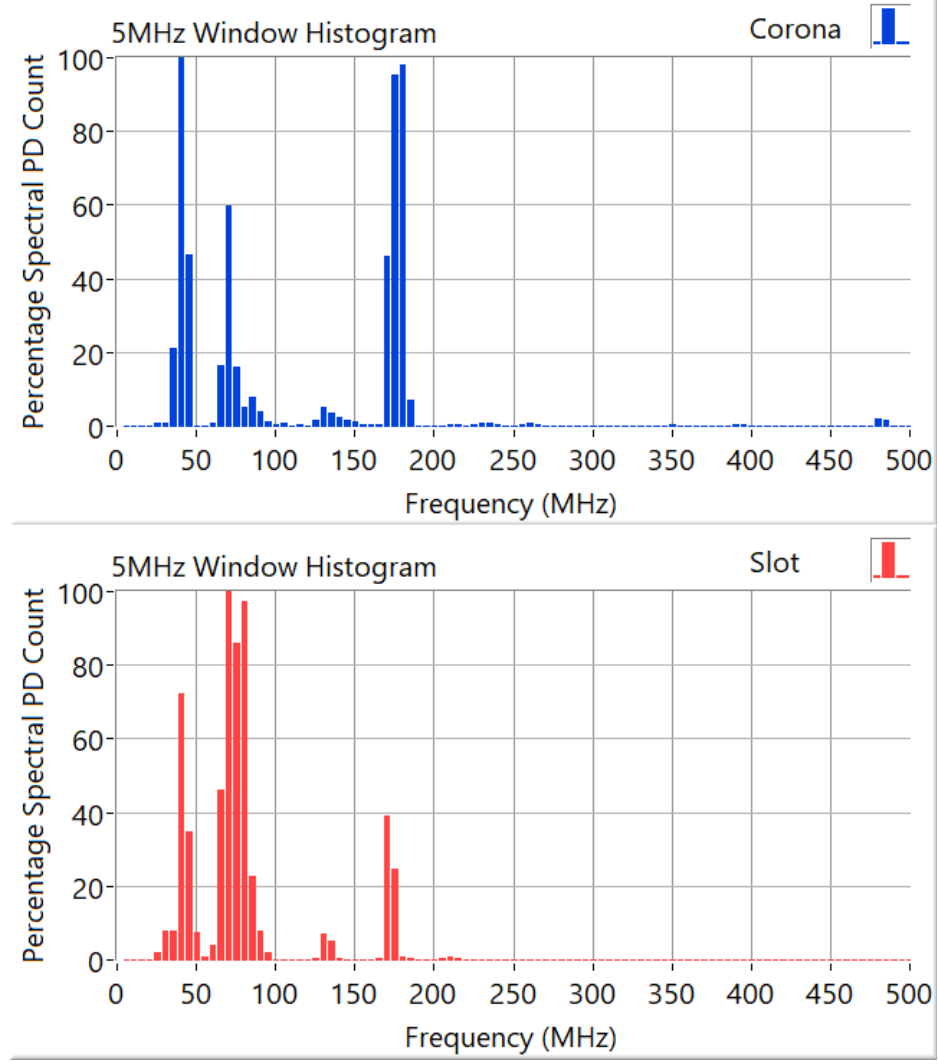

(b)

Figure 8. Spectral histograms of corona and slot PDs: (a) peak amplitude histograms and (b) normalized (percentage) pulse count histograms. 
In order to build FRPD maps, such as the illustrative example of Figure 6, since frequencies at which of PD pulses occur can slightly fluctuate for a given sinusoidal phase, we employed measuring frequency band windows, similarly to the $5 \mathrm{MHz}$ wide windows seen in the histograms of Figure 8. Thus, powers of pulses crossing the power noise threshold in a given measuring frequency window are averaged over time. Furthermore, the number of PD occurrences are individually computed for each frequency window. Therefore, every time a pulse crosses the threshold within a given frequency window, the number of PD occurrences is incremented for that window.

Peak amplitude histograms of Figure 8a show that spectral amplitudes of corona PDs tend to be almost constant over the frequency range shown, while slot PDs amplitudes oscillate over the frequency spectrum, with power peaks considerably higher than the power levels reached by corona PDs. This feature is associated with the electrically closing arcs between the circuit poles during slot PD occurrence. Notice that simultaneous occurrences of corona and slot PDs would be difficult to detect using peak spectral amplitudes. On the other hand, the pulse count histograms in Figure $8 \mathrm{~b}$ show $100 \%$ for the normalized percentage count for slot PD at $70 \mathrm{MHz}$, which corresponds to 451,875 spectral peaks recorded in this frequency window, the counting of which is used as the reference level to set the percentage for the discussed measurements in other frequency bands. The pulse count histogram for corona PD shows $100 \%$ for the normalized percentage count in the $40 \mathrm{MHz}$ window, which corresponds to 394,956 recorded spectral peaks. Thus, corona PDs have higher PD count rates at $40 \mathrm{MHz}$ (with 100\%), $45 \mathrm{MHz}$ (with 46.6\%), $70 \mathrm{MHz}$ (with 59.9\%), $170 \mathrm{MHz}$ (with 46.2\%), $175 \mathrm{MHz}$ (with 95.5\%), and $180 \mathrm{MHz}$ (with 97.9\%). Slot PDs have their higher PD count rates at $40 \mathrm{MHz}$ (with 72.4\%), $45 \mathrm{MHz}$ (with 34.9\%), $65 \mathrm{MHz}$ (with 45.9\%), $70 \mathrm{MHz}$ (with 100\%), $75 \mathrm{MHz}$ (with 86.0\%), $80 \mathrm{MHz}$ (with 97.1\%), and $170 \mathrm{MHz}$ (with 39.2\%). Corona discharges tend to have higher counting rates distributed over the spectrum because they occur between the energized electrode and the air, with plasma channel length highly dependent on the 50 or $60 \mathrm{~Hz}$ instantaneous signal amplitude. In contrast, slot PD counting strongly depends on arcs connecting the circuit poles, producing characteristic resonance frequency, which is associated to the length of the produced ionized electric arcs (which tends to be the distance between the poles) and especially on the total length of the metallic parts shortened by the arcs (the lengths of hydro-generator bars [31], for instance). This behavior explains the fact that slot PDs have, in our test measurements, their highest registered PD repetition rate around $70 \mathrm{MHz}$. Complementary Figure 9 shows spectral pulse counting per second, obtained by sampling the spectra 5000 times over $150 \mathrm{~s}$.

An important matter is related to the spectral peaks seen in Figure 5. Although spectra of current PD pulses can be smooth functions, with a certain bandwidth, it is demonstrated in detail in [31], using full-wave simulations (by solving Maxwell's equations numerically), that electromagnetic waves produced by the PD currents resonate inside the winding bar while propagating in the structure, producing resonating electromagnetic fields propagating outwards from the bar. The fundamental resonance frequencies depend on the dimensions of the bar and on the wave propagation velocity inside the structure. In [31], an analytical formula from [32] is also used to confirm the resonance frequencies obtained from the simulations. Higher harmonics are also seen in simulation results and confirmed using the analytical formula. This physical phenomenon produces electric field spectra with well-defined spectral peaks, due to the influence of the bar and of spectral band of the PD current pulse. It is interesting to see the effect of increasing the pulse rise time in [31], which reduces spectral amplitudes in higher frequencies, but resonance peaks are still seen if excitation pulse has energy levels at those higher frequencies. The mentioned resonance effects are stronger in slot PDs because metallic slot structure reflects an electromagnetic field, forcing it to penetrate the bar (deterioration of semiconductive coating facilitates wave penetration). Corona PDs can occur in a face of the bar with no contact with slot structure, and, thus, a smaller part of electromagnetic wave power refracts into the bar, creating different spectrogram patterns (see Figure 5a,b). 

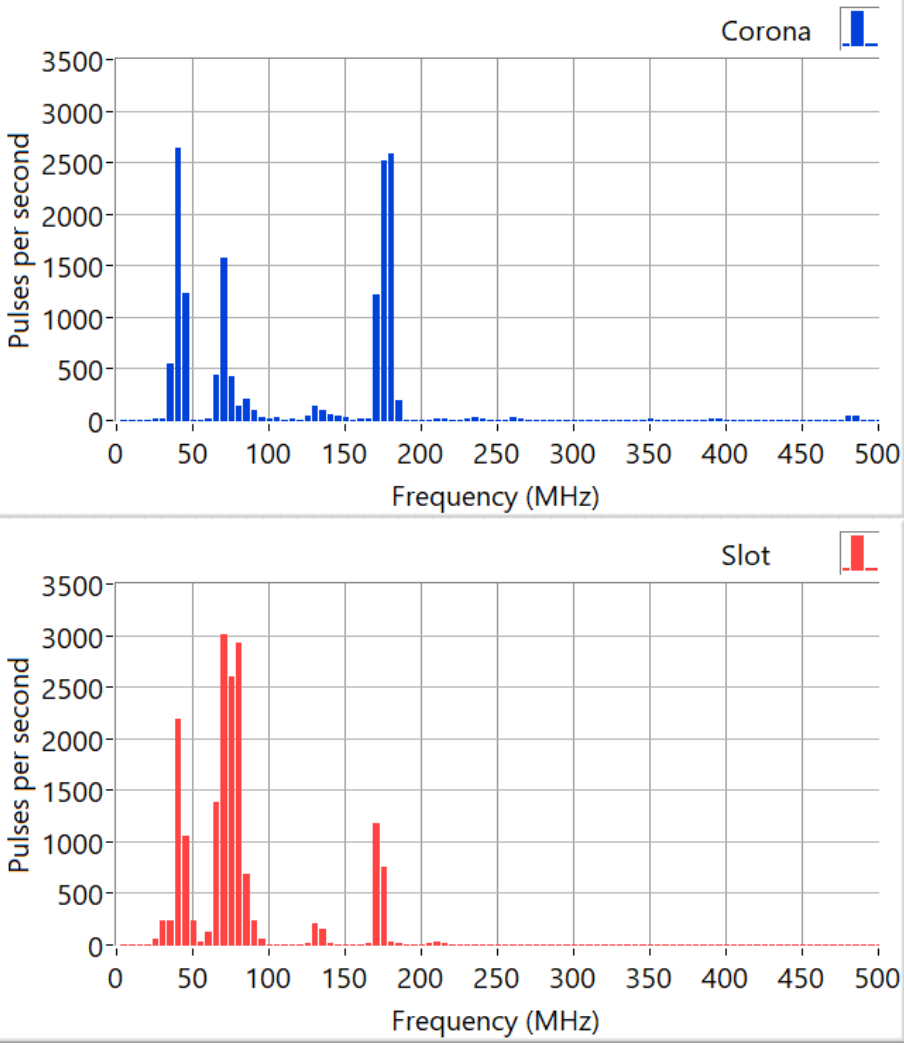

Figure 9. Spectral pulses per second registered in this work, during $150 \mathrm{~s}$, for corona and slot PDs.

Temporal analysis of simulated PD radiated signals in [31] show that resonating frequencies may occur asynchronously, as transient signals clearly contain different frequencies at different time windows. This feature was confirmed in this work in the laboratory, when radiated signals' spectra were measured 5000 times over $150 \mathrm{~s}$. Spectral peaks occur asynchronously, with particular repetition rates over time.

Thus, the peaks seen in Figure 5 are due the electromagnetic phenomenon just described. Notice that the spectrum is actually continuous, but amplitudes attenuated by the bar (at non-resonating frequencies) are not visible in Figure 5 because they are mixed with environmental noise.

Thus, an advantage of using the proposed FRPD methodology is that it allows the identification of classes of partial discharges occurring concurrently, especially those of which PRPDs or spectra are difficult to distinguish when they are superimposed, as is the case involving concurrent corona and slot PDs.

\subsection{Computer Implementation of FRPD}

Computer implementation of FRPD is based on two one-dimensional arrays: one array for PD count and another for average PD amplitude, computed using peaks crossing the power threshold for each frequency window individually. The dimensions of both arrays are coincident with the number of employed frequency windows.

The measurements of spectra of PD signals are made in time windows of $5 \mathrm{~ms}$ with a spectrum analyzer logically connected to a personal computer. The FRPD maps are formed with six frequency sub-bands, namely $7-20 \mathrm{MHz}, 20-100 \mathrm{MHz}, 100-200 \mathrm{MHz}$, 200-300 MHz, 300-400 MHz, and 400-500 MHz. For each frequency sub-band, 5000 time windows are acquired and 2000 measuring frequency windows are employed. Thus, for the $7 \mathrm{MHz}-20 \mathrm{MHz}$ sub-band, measuring frequency windows are $6.5 \mathrm{kHz}$ wide. In the $20 \mathrm{MHz}-100 \mathrm{MHz}$ band, measuring frequency windows are $40 \mathrm{kHz}$ wide. Finally, in the sub-bands $100-200 \mathrm{MHz}, 200-300 \mathrm{MHz}, 300-400 \mathrm{MHz}$, and $400-500 \mathrm{MHz}$, measuring frequency windows are $50 \mathrm{kHz}$ wide. 
As previously discussed, the methodology consists of detecting the PD pulses in a frequency spectrum exceeding the noise power threshold (in $\mathrm{dBm}$ ). The threshold is determined to minimize the influence of background noise. The threshold is determined automatically and experimentally by the software. First, the noise signal is measured and divided into 10 frequency sub-bands. Each of them has its average power value calculated within $10 \mathrm{~s}$. The highest average power is selected, and then, the noise threshold corresponding to $108 \%$ of the highest calculated average noise power is established.

During PD measurements, when any pulse in the spectrum exceeds the threshold, the pulse amplitude value, its frequency value, and the number of times that pulses occurred will be stored for each of the measuring frequency windows. Once the measurements and data recordings are complete, the total count of the occurrences of the spectral PDs pulses is determined, and the average PD amplitude is calculated for each measuring frequency window.

More specifically, for each measuring frequency window $f_{w}$, the number of pulse occurrences $P_{o}\left(f_{w}\right)$ is divided by the highest number of pulse occurrences found, $\max \left\{P_{o}\right\}$, which is obtained among all the sub-bands. Then, the so-called percentage PD count function $P_{c}\left(f_{w}\right)$ is recorded, of which values are mapped by using a color scale. Thus, the function $P_{c}\left(f_{w}\right)$ is given by

$$
P_{c}\left(f_{w}\right)=\frac{P_{o}\left(f_{w}\right)}{\max \left\{P_{o}\right\}} \times 100 \% .
$$

Finally, results obtained from (3) are used to plot FRPDs such as that in Figure 6 and normalized (percentage) pulse count histograms such as those in Figure 8b. Thus, 100\% pulse count indicates the frequency window in which maximum $P_{o}$ was obtained in a given FRPD map or pulse count histogram. The algorithm for the proposed methodology is graphically shown in Figure 10.

By analogy with the PRPD (Phase Resolved Partial Discharge) method [17], which associates the PD peak occurrence with the sinusoidal signal phase, we may say that this new spectral statistical map is a Frequency Resolved Partial Discharge map because, with the presented technique, maps are assembled from PD peak counting derived from time evolving frequency spectrum of PD signals. The main advantage of FRPD over PRPD is the fact that FRPD can be easily used to detect more than one concurrent PD type, even with overlapping PRPDs and spectra, as is shown experimentally in the next sections. 


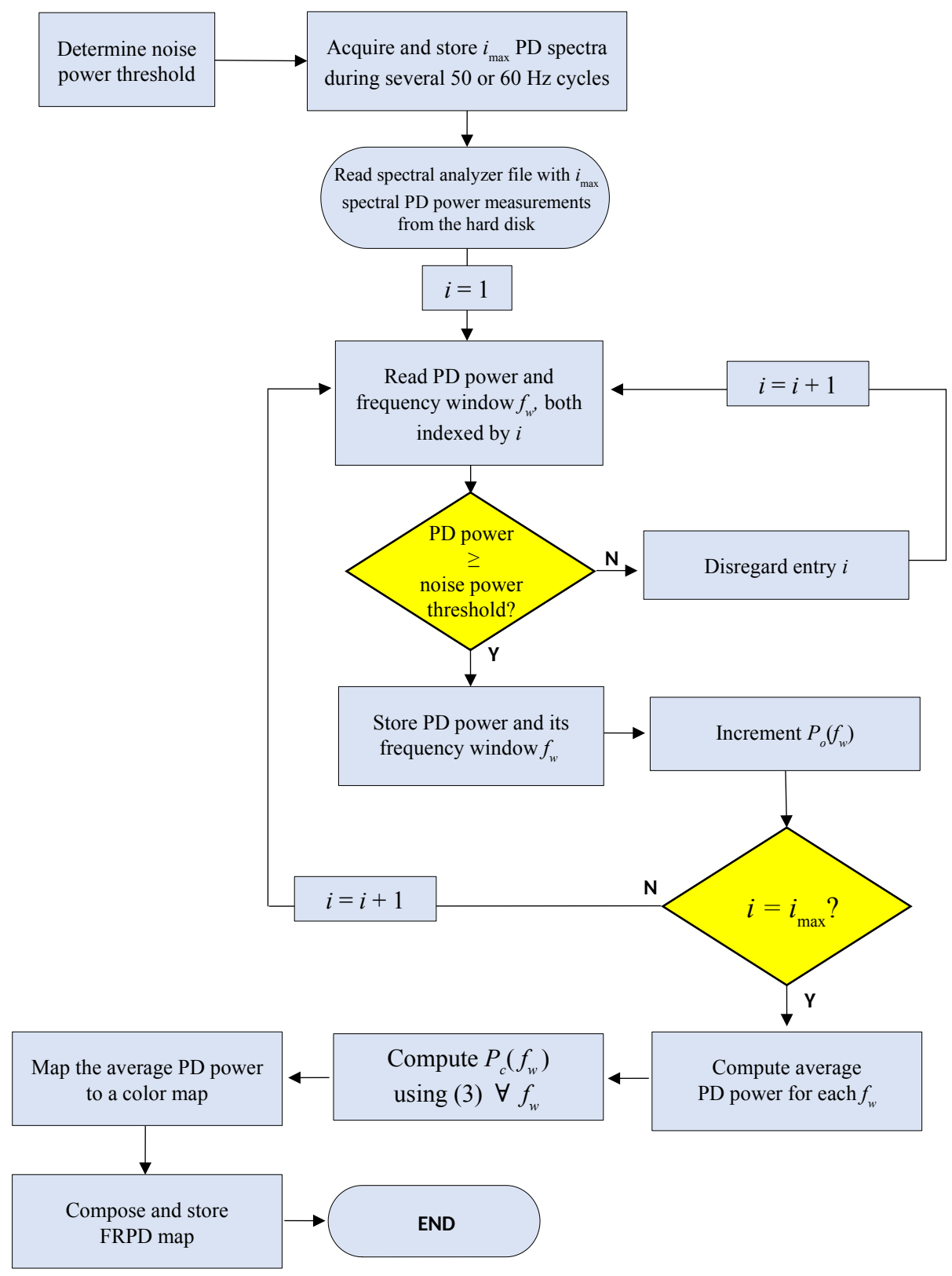

Figure 10. Algorithm for the proposed FRPD methodology. In this work, $i_{\max }=5000$.

\section{Experimental Measurements and Results}

\subsection{The Designed Golden Ratio Patch Antenna}

Since partial discharges radiate high-frequency electromagnetic signals, recently, microstrip patch antennas (consisting of a dielectric substrate with a face containing a specifically designed metallic patch and a ground plane of metal deposited on the opposite substrate face) have been used for PD detection [20,33-35]. Ultra-wide-band (UWB) patch antennas for partial discharge detection were proposed in [33] (0.5 GHz to $1.5 \mathrm{GHz})$, ref. [34] (0.305 GHz to $1.495 \mathrm{GHz})$, and [35] (0.35 GHz to $1.38 \mathrm{GHz})$. An appropriate band for detecting PD radiation is from $0.3 \mathrm{GHz}$ to $1.5 \mathrm{GHz}$ [35]. However, PD frequencies over $1.5 \mathrm{GHz}$ were found in [20].

The well-known golden ratio can be observed not only in biological systems such as shell formation and flower petal formation, but also at the atomic scale in the magnetic resonance of spins in cobalt niobate crystals [36]. In telecommunications engineering, the golden ratio has been used in antenna design $[37,38]$. Therefore, in this work, we design a golden ratio patch antenna operating between $0.33 \mathrm{GHz}$ and $2.02 \mathrm{GHz}$. The patch is 
formed by three circles that are centered on the borders of smaller intercepting circles, such as is illustrated by the proposed design shown in Figure 11. For the designed antenna, $R_{1} / R_{2}=R_{2} / R_{3}=r_{a}=1.618$, which is the golden ratio. The developed antenna has been used to receive high frequency electromagnetic PD signals in this work. Figure 12a properly illustrates the antenna being used in our labaratorial setup.
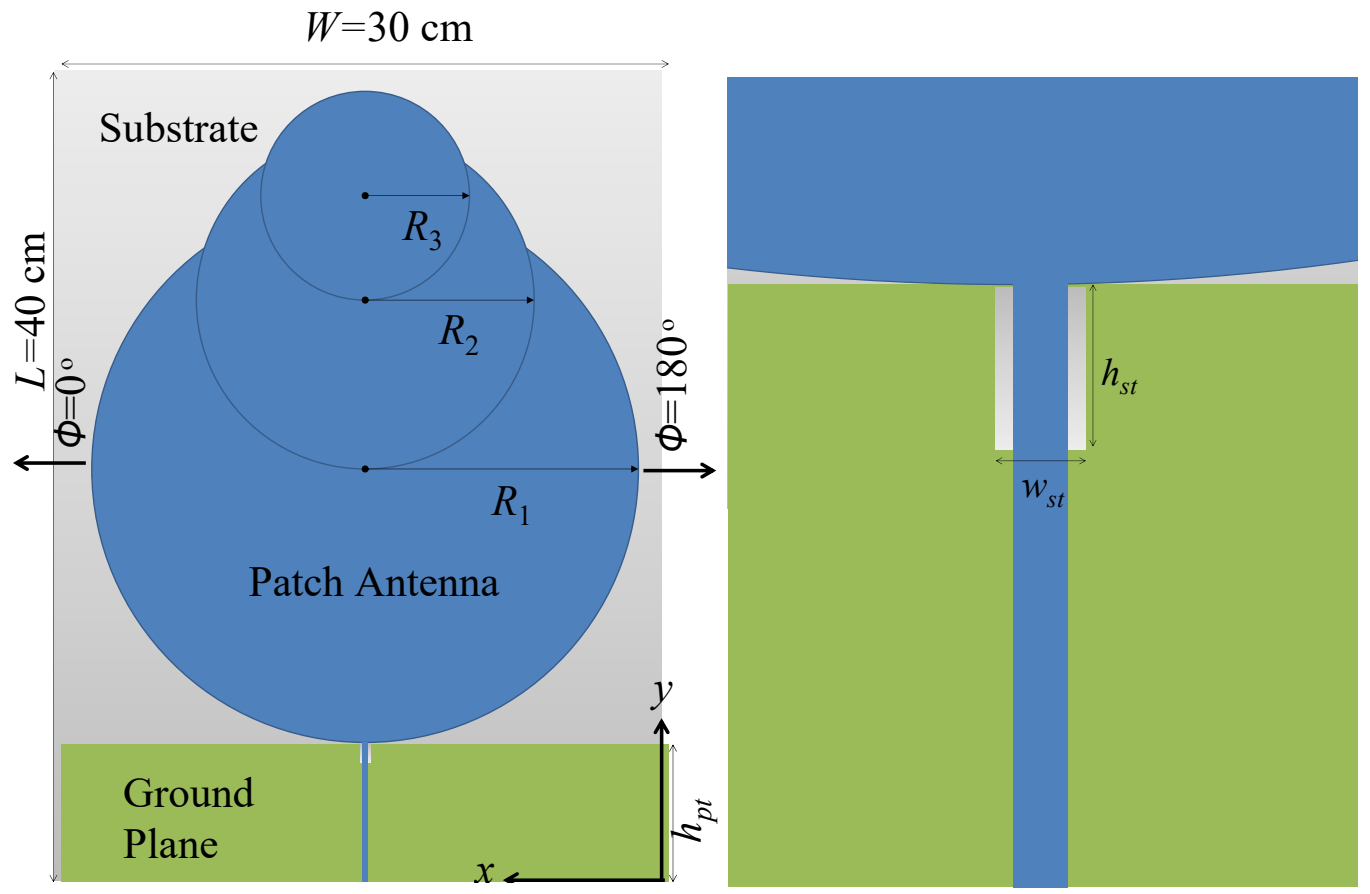

(a)

(b)

Figure 11. Preliminary antenna model simulated using COMSOL Multiphysics: (a) overview and (b) details of the ground plane slot (the ground plane and patch are on opposite sides of the substrate).

For sake of comparison to other antennas, spiral antennas are low-gain wide-band antennas [39], while rod antennas can be high-gain narrow-band devices (e.g., Yagi antennas) [40]. Spiral antennas (with reflector) can achieve $2 \mathrm{dBi}$ gain [39] and Yagi antennas can easily achive $12 \mathrm{dBi}$ or more [40]. Our patch antenna lies in between the two: it is a wide-band antenna with the moderate maximum gain of approximately $3.8 \mathrm{dBi}$ at $450 \mathrm{MHz}$. Further details, parameters, and engineering characteristics of the device are described in detail in the Appendix A.

\subsection{Laboratory Setup}

The designed golden ratio patch antenna was used to perform high-frequency PD measurement with the setup illustrated by Figure 1. In this work, the process of measuring PD signals to produce FRPD maps involved the following instruments and apparatus: a spectrum analyzer, the patch antenna, hydro-generator stator bars, a $60 \mathrm{~Hz}$ high voltage source, and a laptop computer with the measurement and analysis software developed in this work. The experimental setup can be seen in Figure 12a. 


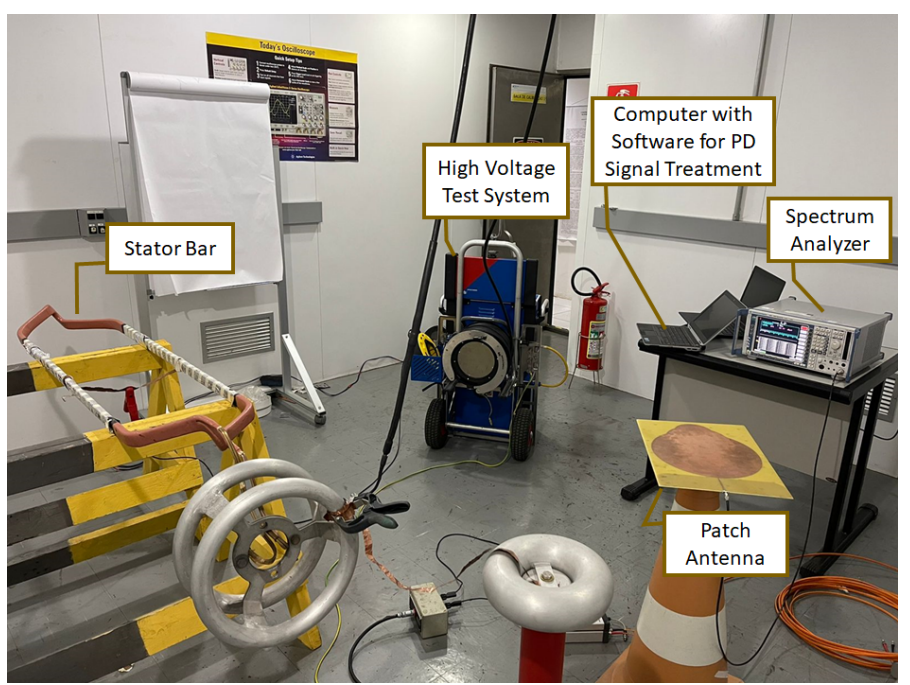

(a)

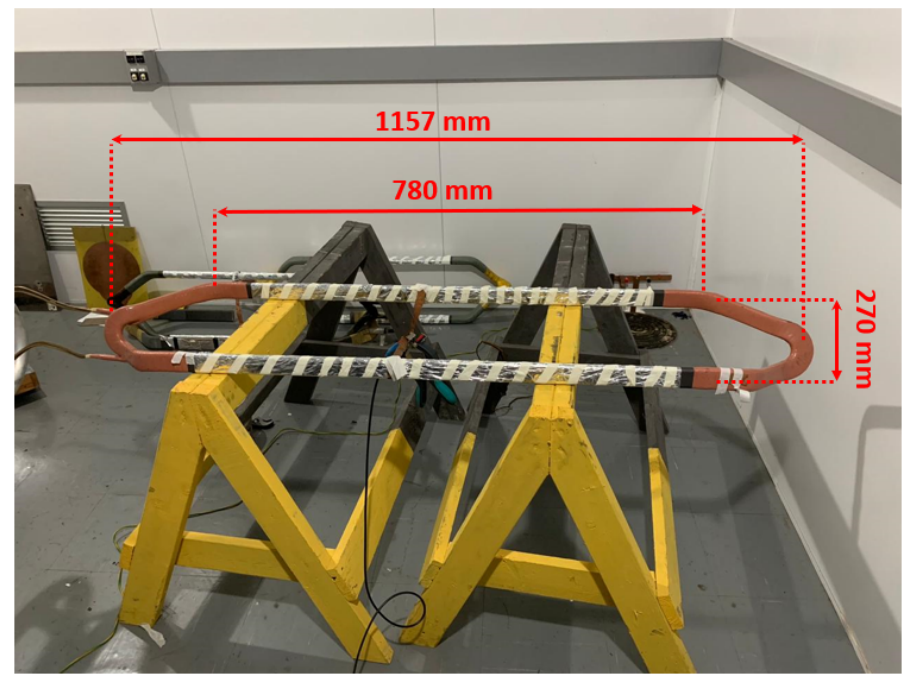

(b)

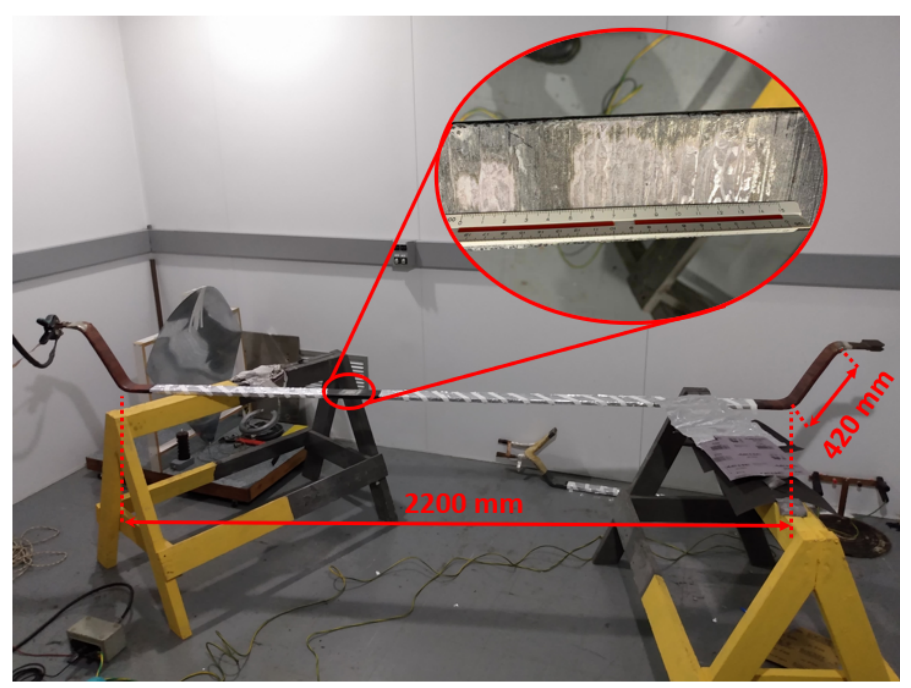

(c)

Figure 12. Cont. 


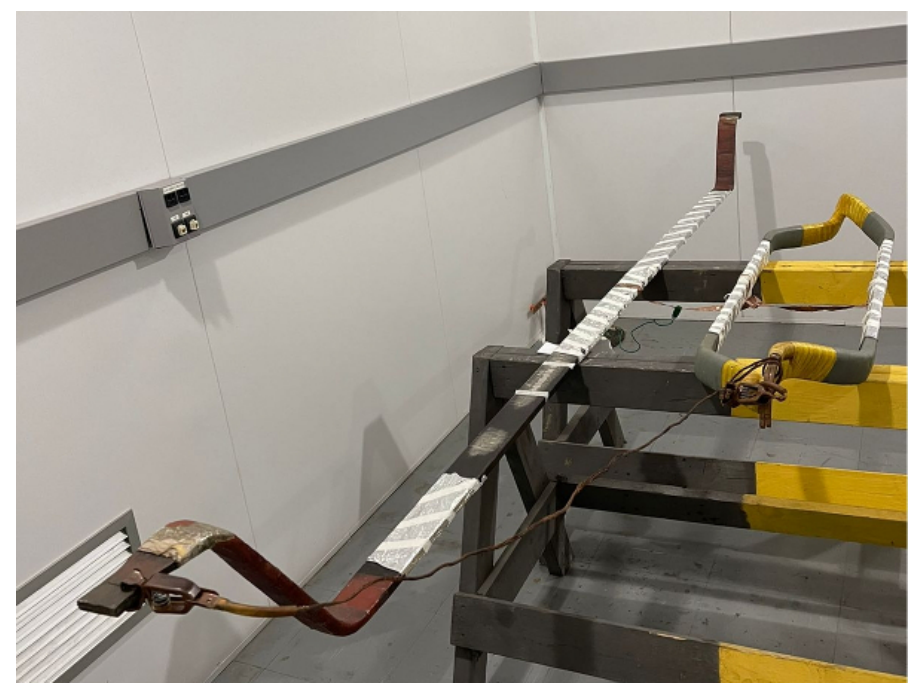

(d)

Figure 12. The measurement setup: (a) overview of the measurement setup for acquiring high frequency PD radiated signals, (b) General Electric bar from Curuá-Una hydroelectric power plant (with corona PDs), (c) Jeumont-Schneider bar from Tucuruí hydroelectric power plant (with slot PDs), and (d) parallel-connected bars for producing concurrent corona and slot PDs.

The hydro-generator bars were energized with $12 \mathrm{kV}$ by the $60 \mathrm{~Hz}$ high voltage system. This voltage level was sufficient to capture the PD signals from the bars using the patch antenna. The patch antenna was connected to the spectrum analyzer using a $50 \Omega$ impedance coaxial cable. PD spectral signals, acquired over time, were registered by the developed software in which the proposed FRPD methodology is implemented.

For the measurement of the corona discharges, a bar with mica insulation from the Curuá-Una Hydroelectric Plant (Brazil) was used, which is shown in Figure 12b. For the slot discharges, a bar with mica insulation from the Tucuruí Hydroelectric Plant (Brazil) swas used, which can be seen in Figure 12c. The slot defect produced in the bar is highlighted in Figure 12c. The measurements of concurrent corona and slot PD discharges were performed by implementing a parallel circuit connection between the Curuá-Una and the Tucuruí Hydroelectric Plant bars, as is seen in Figure 12d.

\subsection{Results}

Figure 13 shows PRPDs obtained in this work for the experiments of Figure 12. The measured PRPDs of corona, slot, and the simultaneous corona and slots PDs are shown by Figure 13a-c, respectively. Note that PRPD profiles shown in Figure 13a,b are compatible with standardized PRPD patterns in [6] for corona and slot PDs, respectively, validating our experimental PD sources. By comparing Figure 13a,b, we can see that corona and slot PDs occur practically on the same phase ranges for both the positive and the negative $60 \mathrm{~Hz}$ semi-cycles. For this reason, concurrent corona and slot PRPDs overlap each other in Figure 13c, as one would expect. Thus, distinguishing these defects patterns by inspecting solely Figure 13c is a remarkably difficult task. 


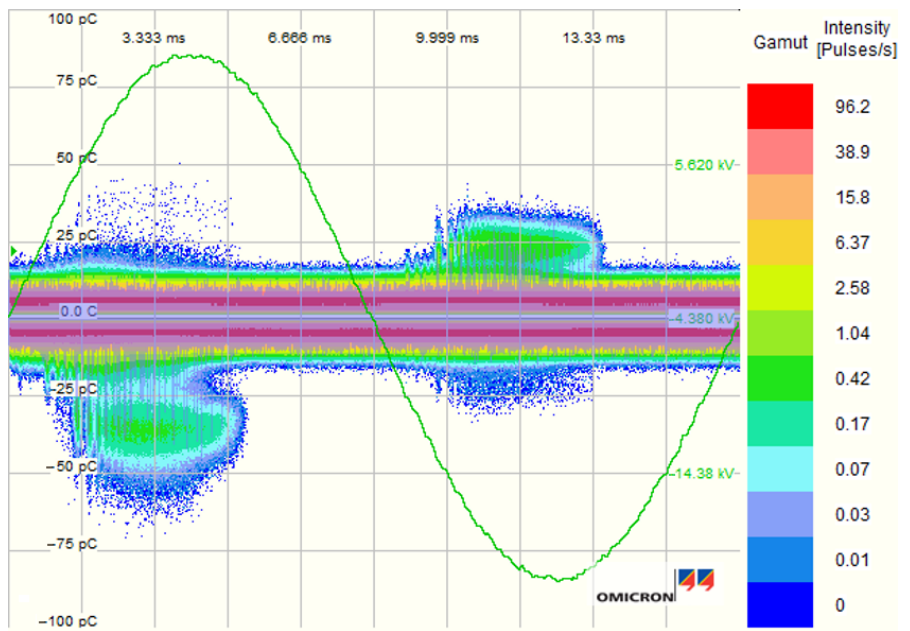

(a)

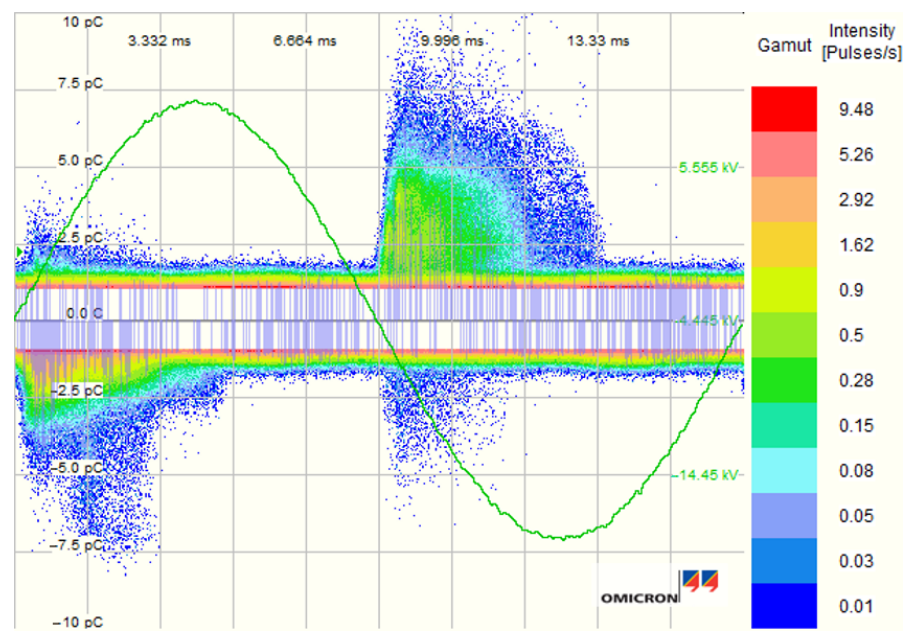

(b)

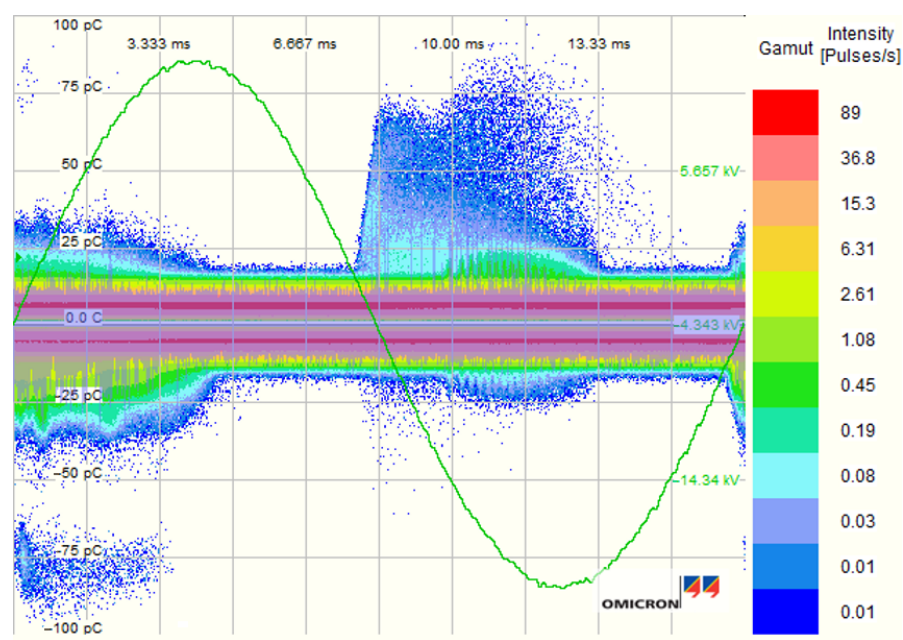

(c)

Figure 13. PRPDs experimentally obtained in this work for: (a) corona (12 kV-General Electric bar), (b) slot (10 kV-Jeumont-Schneider bar), and (c) simultaneous corona and slot (12 kV) obtained usingboth bars. 
The situation is different when FRPDs are used for identifying the measured corona and slot PDs. FRPDs seen in Figure 14 show spectral counting computed from 0 to $500 \mathrm{MHz}$ for different cases: corona discharges in Figure 14a, slot discharges in Figure 14b, and corona PDs with simultaneous slot PDs in Figure 14c. In order to quantify background noise and to map radiated signals from the high voltage source, we have registered the background noise with non-energized and energized high voltage power source, as shown by Figure $14 d, e$, respectively.

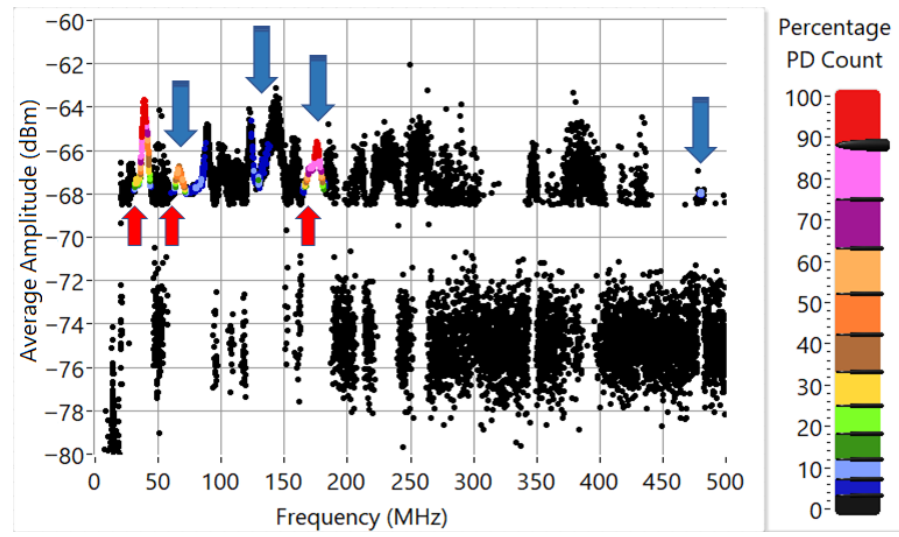

(a)
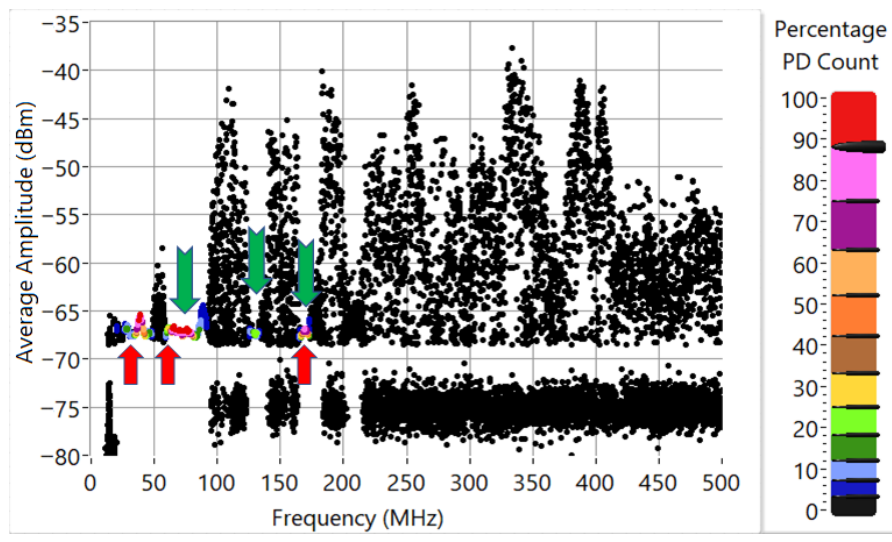

(b)

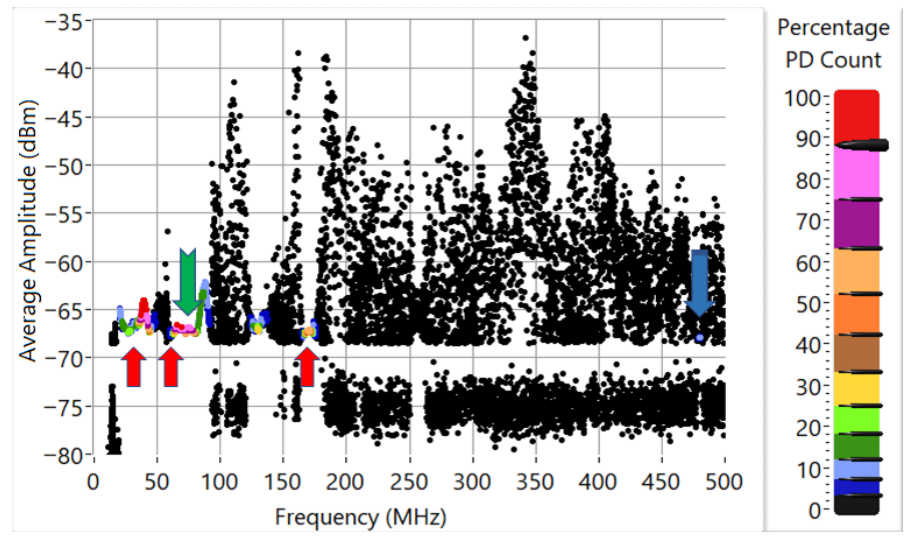

(c)

Figure 14. Cont. 


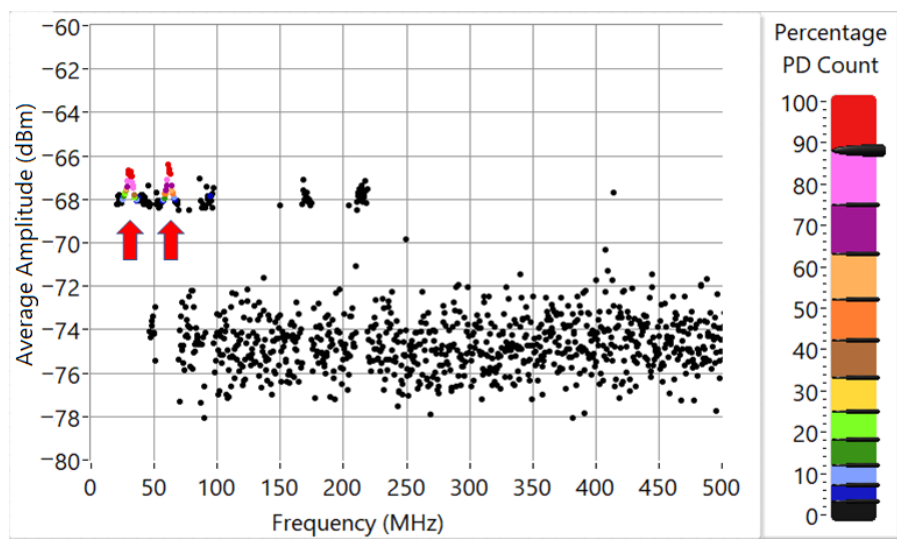

(d)

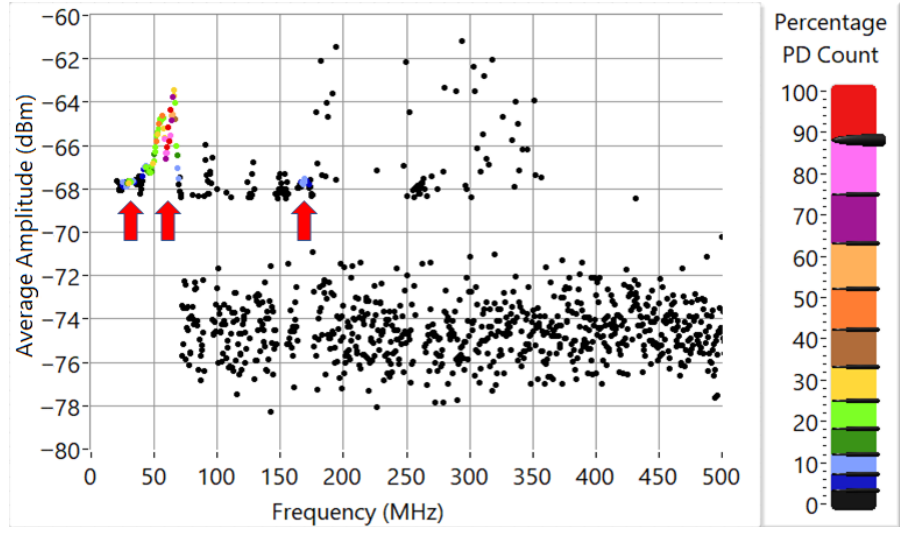

(e)

Figure 14. FRPD obtained from 0 to $500 \mathrm{MHz}$ for different cases: (a) corona PD, (b) slot PD, (c) corona PD with concurrent slot PD, (d) background noise (high voltage power source not energized), and (e) background noise with an energized high-voltage power source.

Once measurements of Figure 14d,e had been made, we considered the noise power threshold of $-68.5 \mathrm{dBm}$ for performing PD measurements. Percentage PD count, which is mapped using a color scale, was calculated with respect to the highest measured counting, which is represented by the color red. Black points indicate low-percentage counting pulses, i.e., up to $3 \%$ of the highest measured counting.

In Figure 14, red arrows (pointing up) indicate high counting noise or high counting signals from the high voltage power source, which are not related to PDs. These external signals were acquired and identified in measurements whose results are shown in Figure 14d,e. In Figure 14a, the blue arrows (pointing down) indicate high-counting spectral regions associated to corona PDs. Similarly, in Figure 14b, the green arrows (pointing down) indicate high-counting pulses associated with slot PDs. Additionally, note that low counting pulses (represented by black points) with amplitudes over $-60 \mathrm{dBm}$ from slot discharges are widely spread across the measuring spectrum, as shown in Figure 14b. This distinct feature is not seen in Figure 14a for corona PDs.

Figure 14c, which was obtained using the measuring setup shown in Figure 12d, shows the simultaneous presence of the corona and slot PDs. Indications are made by following the arrow representations defined above. The low count pulses (black points) are widely spread across the spectrum, indicating the presence of slot discharges in Figure 14c. One may also observe that the spectral regions of high counting pulses corresponding exclusively to corona and slot PDs (around $475 \mathrm{MHz}$ and $75 \mathrm{MHz}$, respectively), which were previously detected in the measurements shown in Figure 14a,b, are also visible and 
properly indicated in Figure 14c. Thus, with the proposed methodology, it is possible to identify, undoubtedly, simultaneous slot and corona PDs.

FRPD maps seen in Figure 15 highlight the results from 7 to $50 \mathrm{MHz}$. Figure 15d,e shows repetitive noise between 25 and $37 \mathrm{MHz}$ and between 41 and $50 \mathrm{MHz}$ (influence of energized power supply), with amplitudes around $-66 \mathrm{dBm}$. Figure 15a, Figure $15 \mathrm{~b}$, and Figure 15c, respectively, show FRPDs for corona, slot, and for corona with slot, with amplitudes reaching $-64 \mathrm{dBm}$. However, frequency bands where high counting corona and slot PDs are observed overlap each other (besides overlapping the high counting band of noise). Thus, in this band, although PDs can be seen standing out from the noise, it is difficult to distinguish concurrent corona and slot PDs.
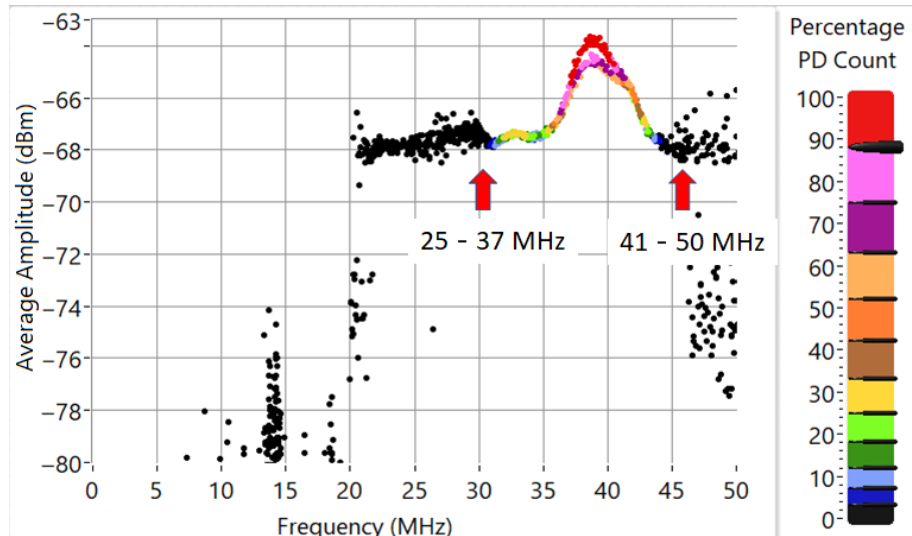

(a)
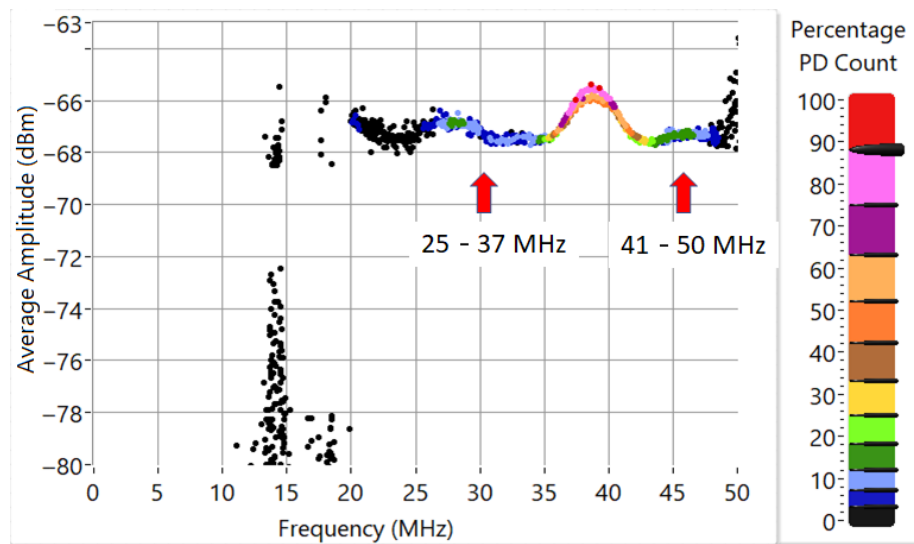

(b)
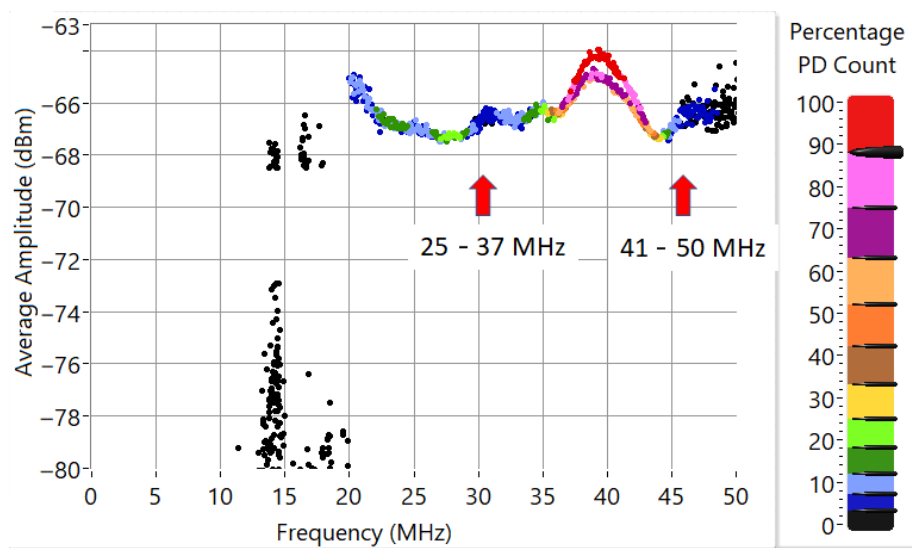

(c)

Figure 15. Cont. 


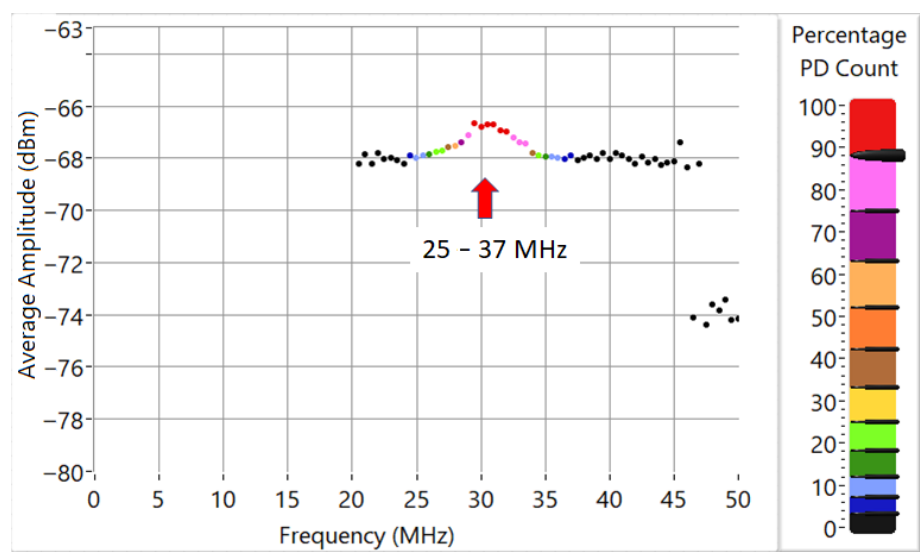

(d)
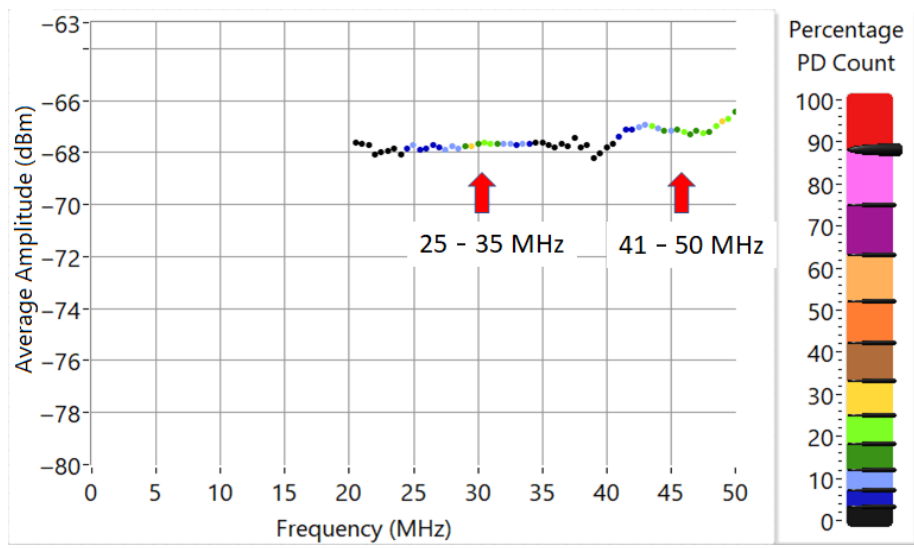

(e)

Figure 15. FRPD for different cases, from 0 to $50 \mathrm{MHz}$ : (a) corona, (b) slot, (c) corona with slot, (d) noise with the power supply not energized, and (e) noise with energized power supply.

Figure 16 shows FRPD results from 50 to $100 \mathrm{MHz}$. Figure 16d,e shows noise between 50 and $70 \mathrm{MHz}$, in such a way that the high voltage source has an important influence on registered counting, with amplitude reaching $-63 \mathrm{dBm}$. Figure 16 a shows corona PDs between 65 and $70 \mathrm{MHz}$, with PD counting up to $63 \%$ and amplitudes reaching $-67 \mathrm{dBm}$ and low counting registered in noise band (50-70 MHz). Figure $16 \mathrm{~b}$ shows slot PDs between 65 and $80 \mathrm{MHz}$, with PD counts up to $100 \%$ and amplitudes that reach $-67.5 \mathrm{dBm}$. In Figure $16 \mathrm{~b}$, the noise region indicated by the red arrow has a low count. Figure $16 \mathrm{c}$ shows simultaneous corona and slot PDs between 63 and $80 \mathrm{MHz}$ with PD counts up to 100\%. The region between 65 and $70 \mathrm{MHz}$ is a region of high PD count shared by corona and slot PDs, as one can see by inspecting Figure 16a,b. The green arrow in Figure 16c highlights the high PD count band, between 70 and $80 \mathrm{MHz}$, where solely slot PDs occur. The band between 80 and $90 \mathrm{MHz}$ is a communal PD band for both phenomena. Thus, in the FRPD of Figure 16c, corona PDs cannot be identified with confidence, but slot PDs can be clearly seen in Figure 16c. 


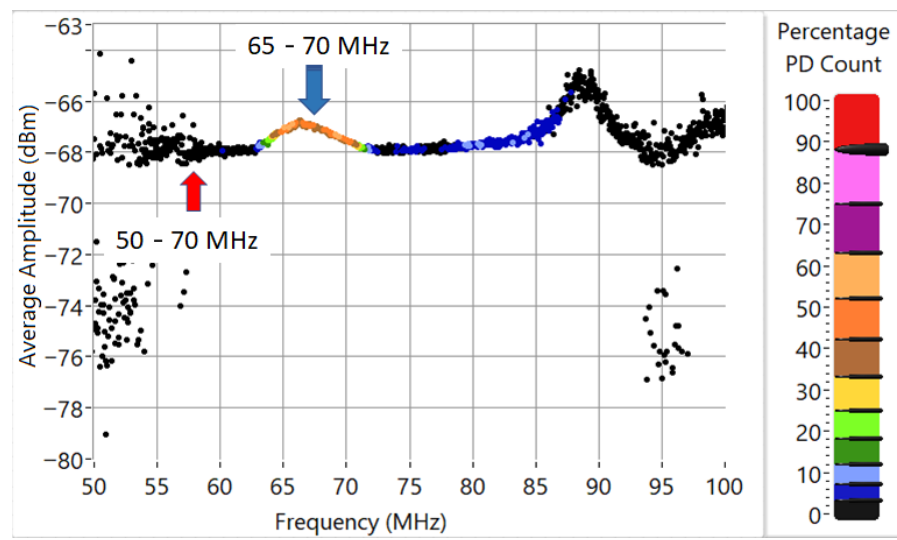

(a)

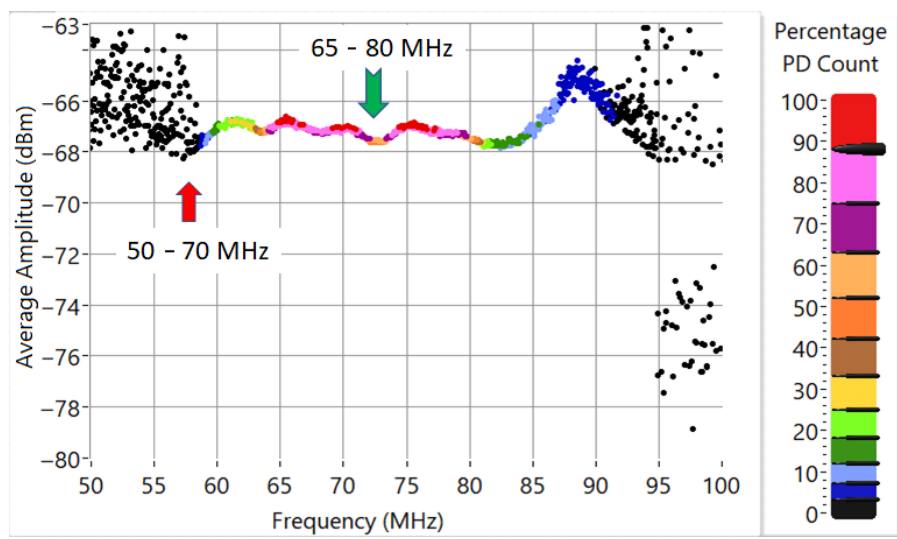

(b)

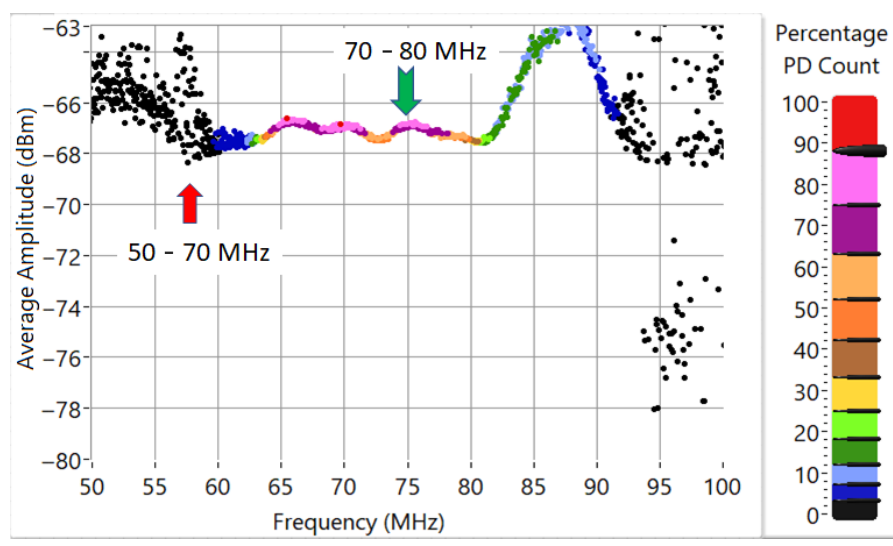

(c)

Figure 16. Cont. 

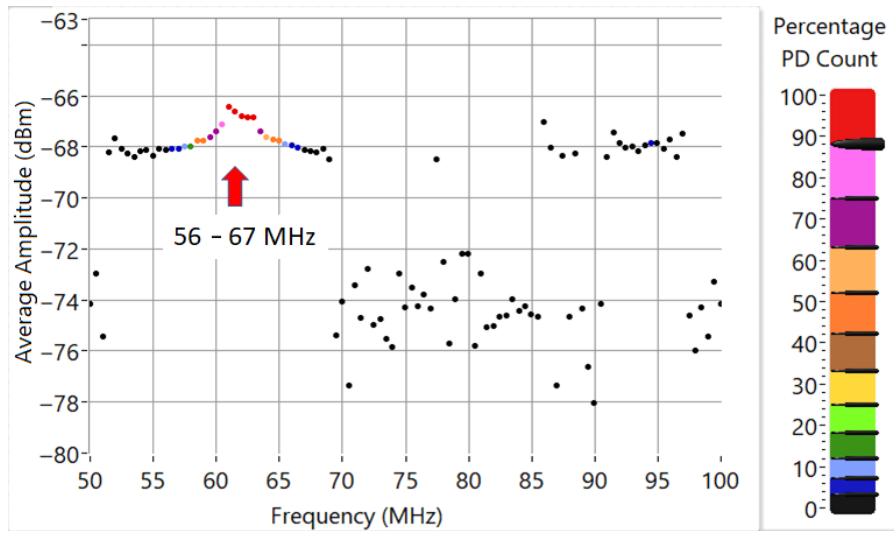

(d)

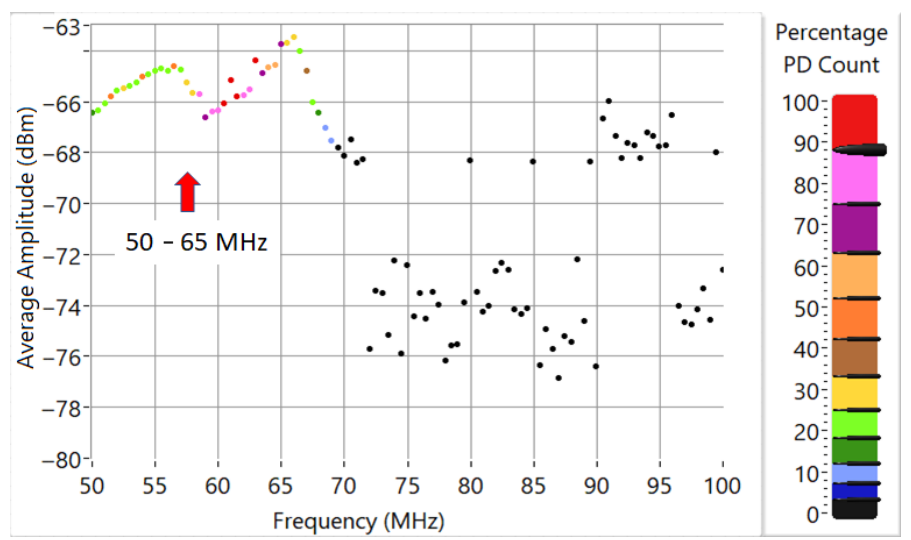

(e)

Figure 16. FRPD for different cases, from 50 to $100 \mathrm{MHz}$ : (a) corona, (b) slot, (c) corona with slot, (d) noise with the power supply not energized, and (e) noise with energized power supply.

FRPDs obtained from 100 to $150 \mathrm{MHz}$ are shown in Figure 17. High counting noise is not seen in Figure 17d,e. Figure 17a shows corona PDs between 124 and $137 \mathrm{MHz}$, with PD counts up to $12 \%$ and amplitudes that reach $-65.5 \mathrm{dBm}$, as the blue arrow indicates. Slot PDs are seen between 125 and $133 \mathrm{MHz}$ in Figure 17b, with PD counts up to 25\% and amplitudes reaching $-67 \mathrm{dBm}$, as the green arrow points out. Figure 17c shows coronas with slot PDs between 125 and $135 \mathrm{MHz}$ with PD counts up to 33\%. In this case, since the corona's and slot's high counting bands completely intercept each other, it is difficult to distinguish the phenomena by inspecting exclusively the $100-150 \mathrm{MHz}$ band. A very similar situation is seen by inspecting the 150-200 MHz range. As Figure 18a,b show, corona and slot high counting bands are completely superimposed on each other, making discrimination of patterns an intricate process when inspecting Figure 18c. 

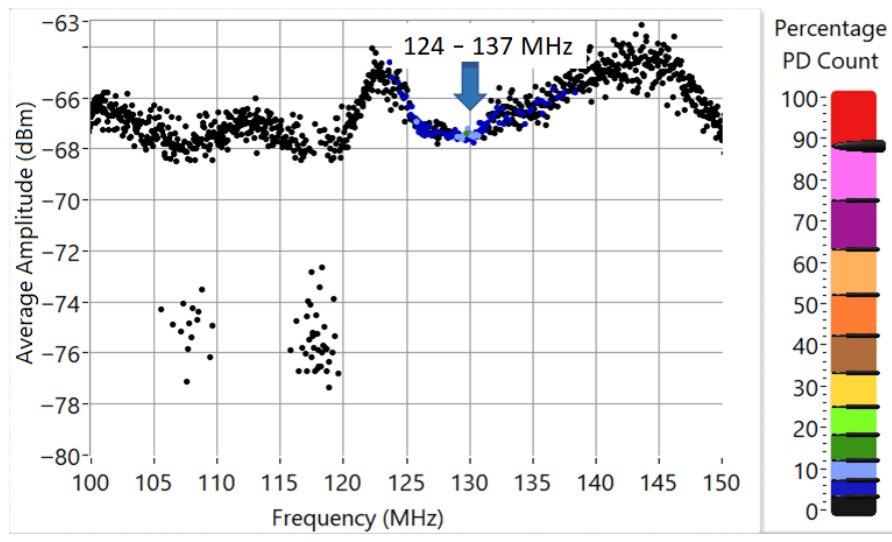

(a)

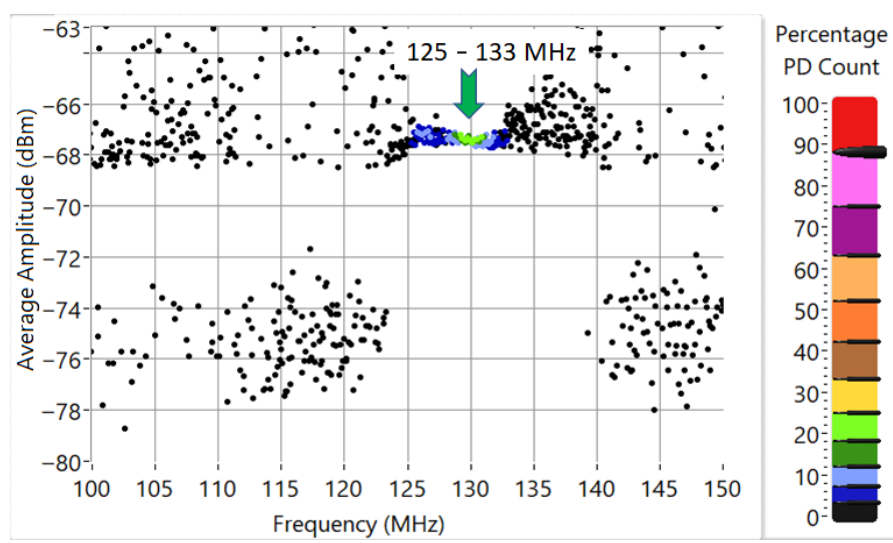

(b)

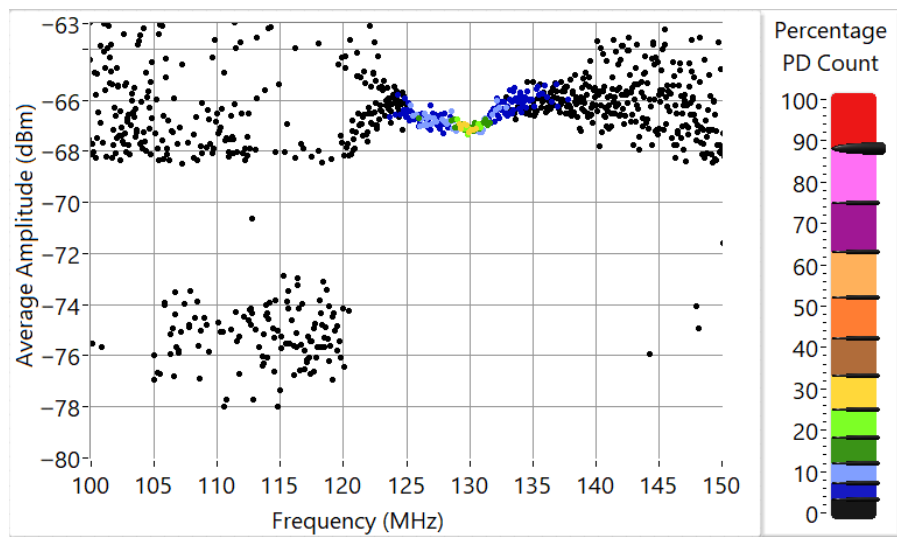

(c)

Figure 17. Cont. 

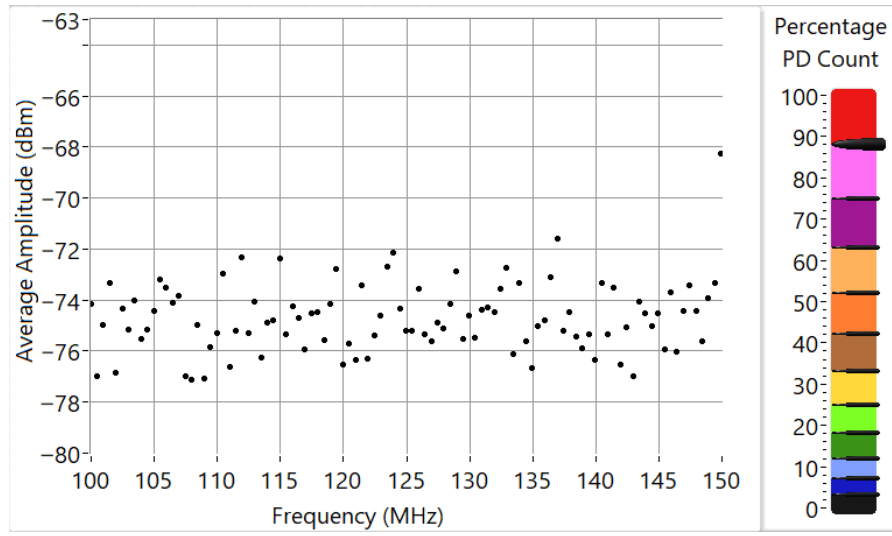

(d)

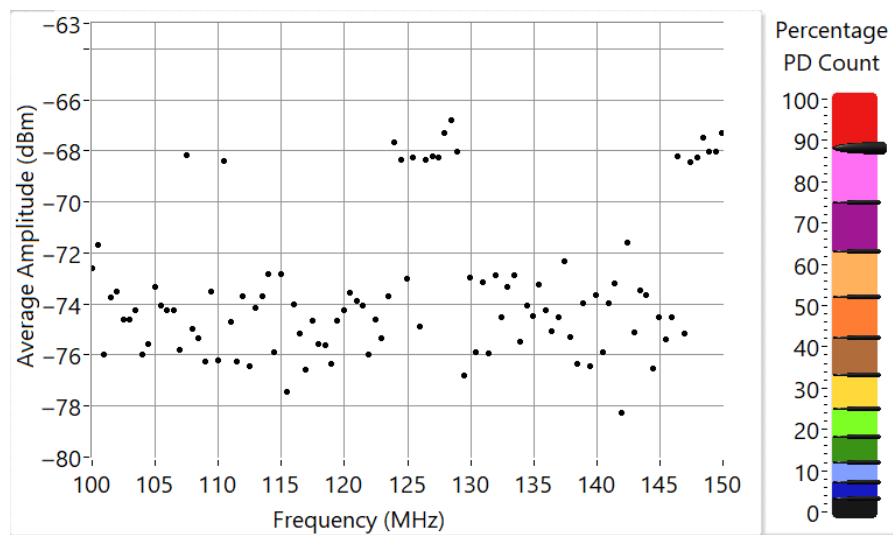

(e)

Figure 17. FRPD for different cases, from 100 to $150 \mathrm{MHz}$ : (a) corona, (b) slot, (c) corona with slot, (d) noise with the power supply not energized, and (e) noise with energized power supply.

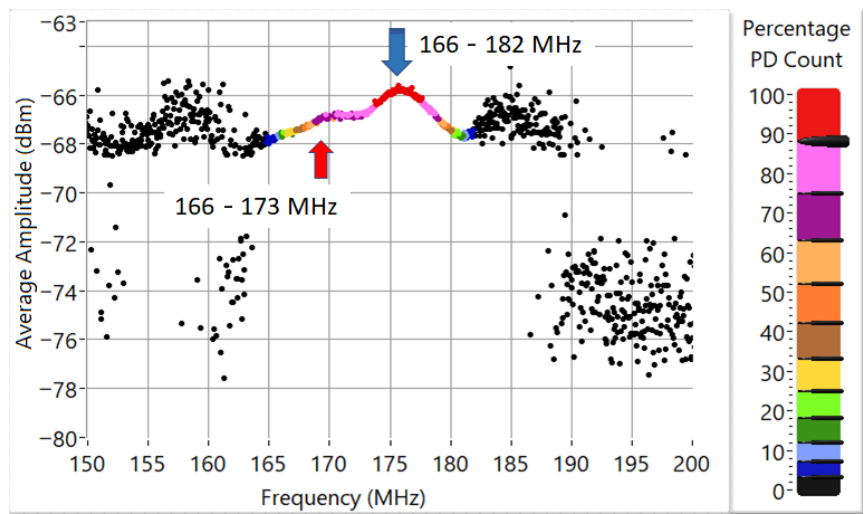

(a)

Figure 18. Cont. 


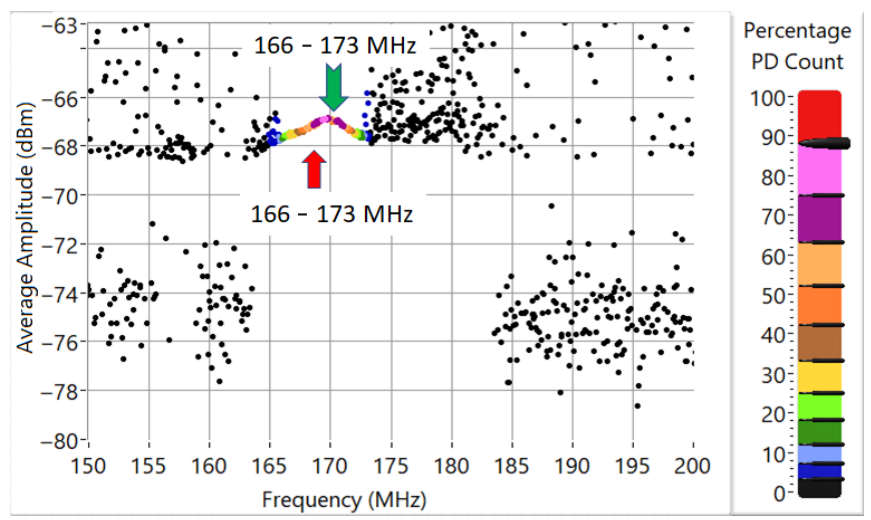

(b)

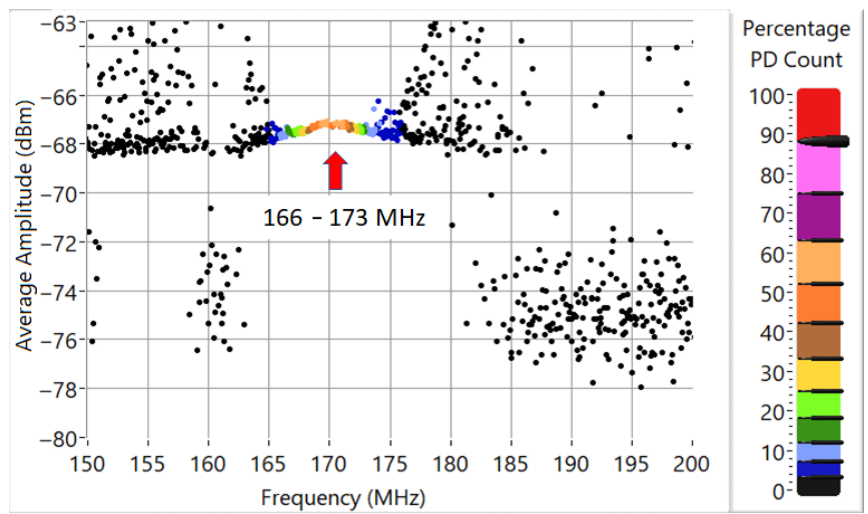

(c)

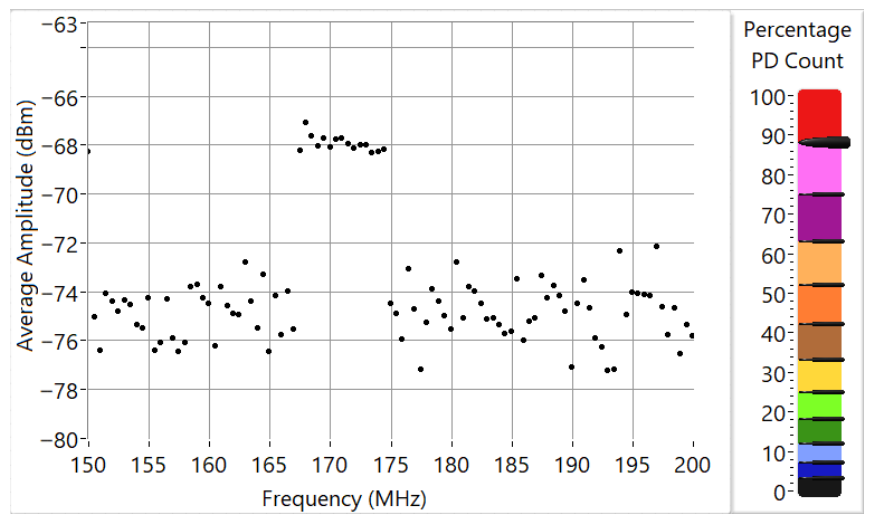

(d)

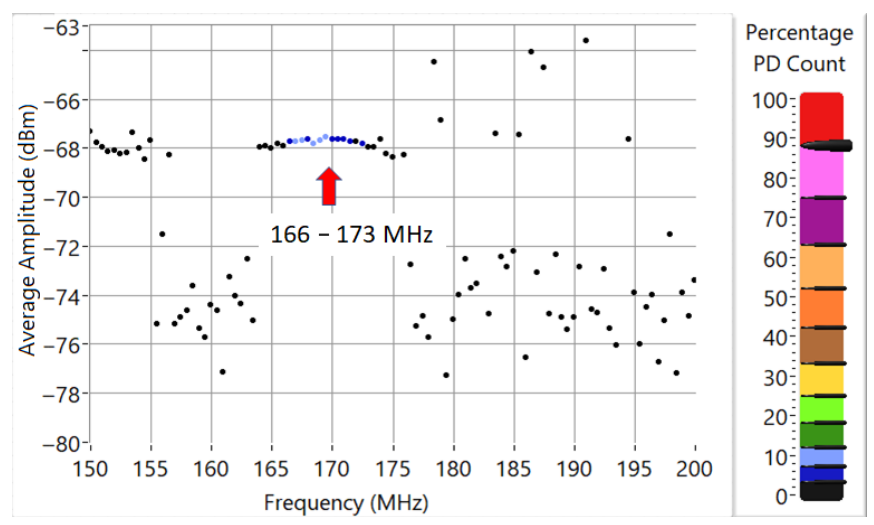

(e)

Figure 18. FRPD for different cases, from 150 to $200 \mathrm{MHz}$ : (a) corona, (b) slot, (c) corona with slot, (d) noise with the power supply not energized, and (e) noise with energized power supply. 
Finally, the FRPDs seen in Figure 19 highlight results from 450 to $500 \mathrm{MHz}$. Figure 19d,e does not show high counting noise in this sub-band. Figure 19a shows corona PDs between 479 and $481 \mathrm{MHz}$, with PD counts up to $12 \%$ and amplitudes that reach $-67 \mathrm{dBm}$, as the blue arrow pinpoints. Figure $19 \mathrm{~b}$ shows occurrence of slot PDs with low PD counts in this sub-band, of which power ranges from -68.75 to $-65 \mathrm{dBm}$. Figure $19 \mathrm{c}$ shows concurrent corona and slot PDs, in which we can see significant PD counts between 479 and $481 \mathrm{MHz}$ (up to $12 \%$ ) with amplitudes reaching up $-68 \mathrm{dBm}$, clearly indicating corona PDs. Furthermore, low count discharges with amplitudes ranging between -68.75 and $-65 \mathrm{dBm}$ are also seen spread across the frequency band in Figure 19c, suggesting the concurrent influence of slot discharges. Therefore, one can properly identify corona and slot PDs in the 450-500 MHz band by inspecting the FRPD map in Figure 19c.

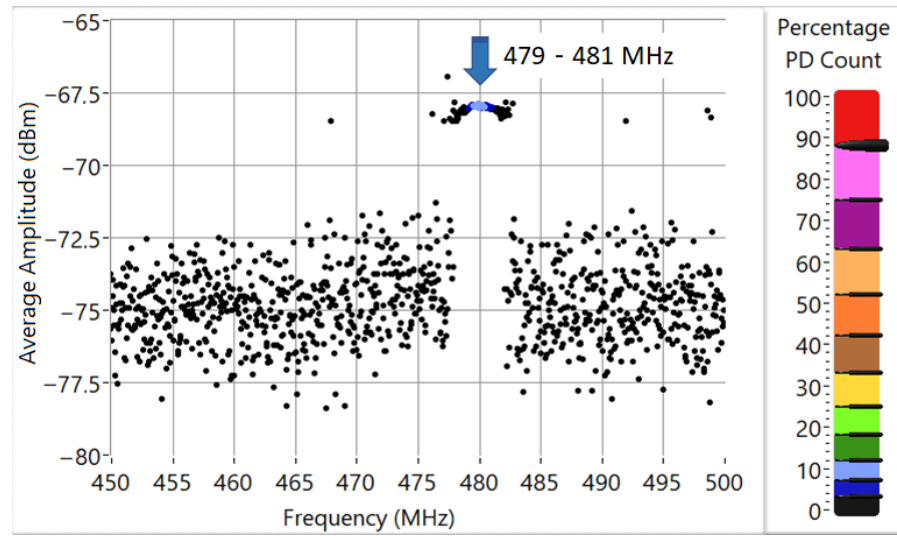

(a)

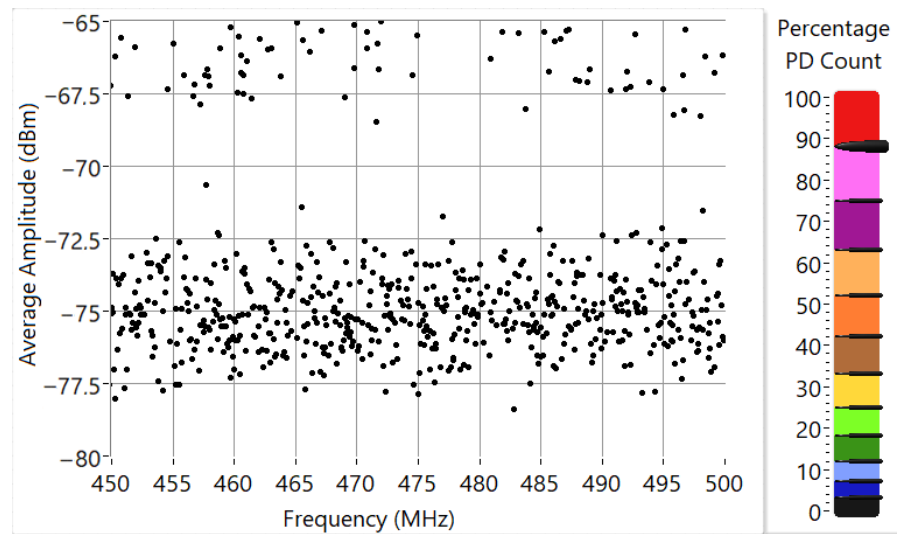

(b)

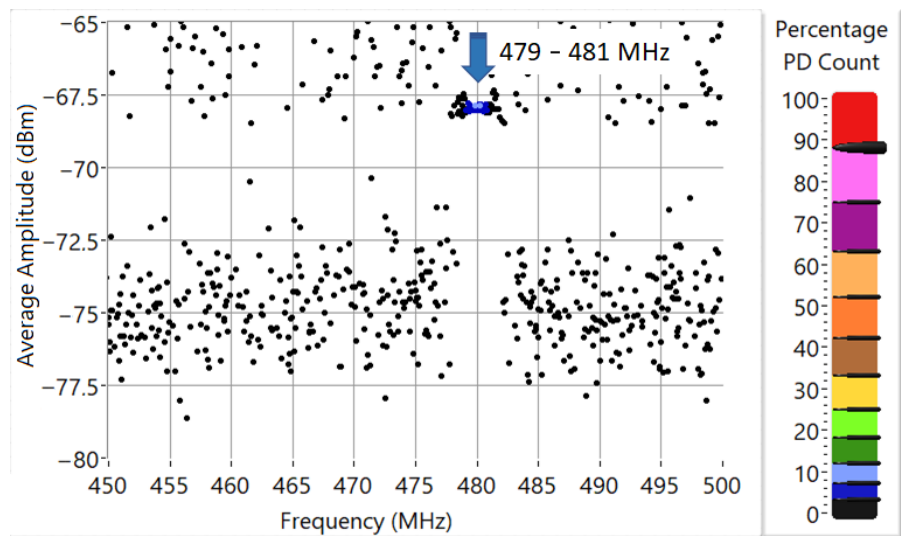

(c)

Figure 19. Cont. 


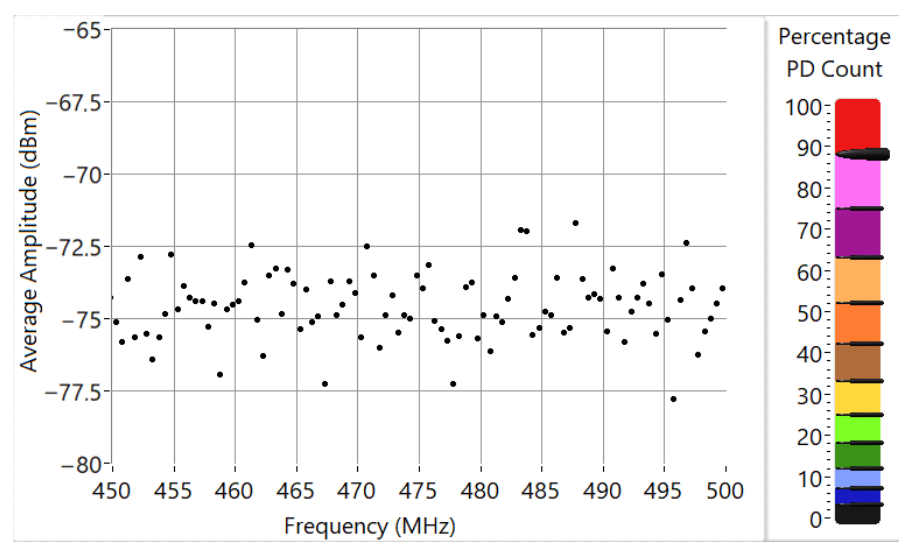

(d)

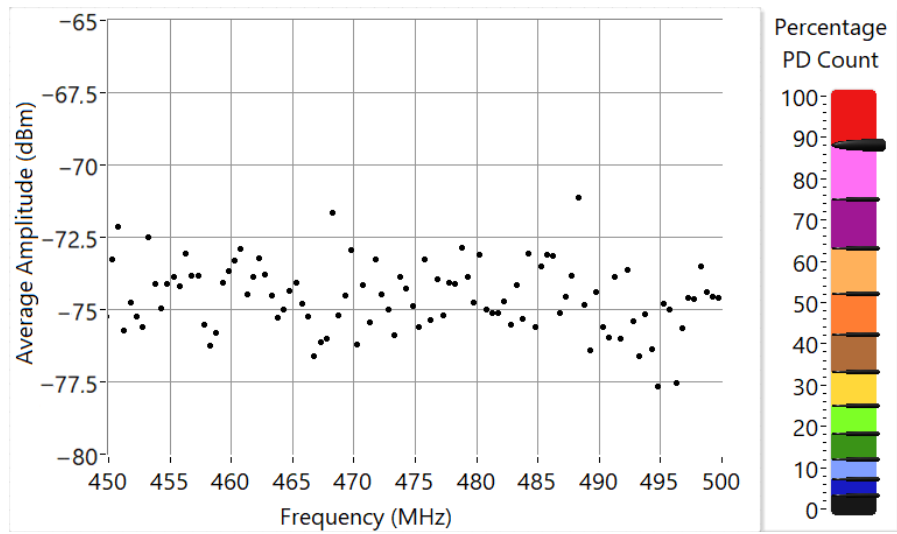

(e)

Figure 19. FRPD for different cases, from 450 to $500 \mathrm{MHz}$ : (a) corona, (b) slot, (c) corona with slot, (d) noise with the power supply not energized, and (e) noise with energized power supply.

\section{Conclusions}

This paper proposes a new methodology for spectral statistical analysis of partial discharge pulses: frequency resolved partial discharge (FRPD). The proposed methodology is based on counting pulses in spectral domain (which evolves over time), allowing the identification of faults that give rise to discharges, even when the signals occupy shared frequency bands and communal phases of the sinusoidal high voltage excitation signal.

In order to measure the electromagnetic signals radiated from partial discharges, a patch antenna was designed, built and employed in laboratory measurements. The antenna was connected to a RF frequency analyzer. Besides that, a software was developed to analyze the discharge signals using the proposed FRPD methodology. The measurements were made in an electromagnetically shielded room, in a high-voltage laboratory, with two bars from hydro-generator stators. One of the bars has a confirmed corona fault and the other one has certified slot PDs, as measured PRPDs show. In order to obtain corona and slot PDs simultaneously, the two bars were electrically connected in a parallel circuit configuration.

With the proposed methodology, one can generate a FRPD graph showing percentage pulse count rates distributed over the frequency-amplitude plane. Thus, specific bands display high counting PDs for each type of partial discharge source, and each type has its own spectral PD counting map. As was shown experimentally in this work, this feature allows the proper identification of simultaneous PD classes with overlapping PRPDs and spectra, such as corona and slot PDs. In particular, the band from $450 \mathrm{MHz}$ to $500 \mathrm{MHz}$ seems to be important for undoubtedly detecting corona and slot FRPD signatures. Since the proposed FRPD methodology seems to be a strong complement for previously 
developed techniques, such as PRPD and classical spectral analysis, i future works, further investigation and possibly standardization of FRPDs are suggested for all known classes of PD sources in rotating machines and other HV systems.

Author Contributions: Conceptualization, R.M.S.d.O.; Methodology, R.M.S.d.O., A.J.C.S. and J.A.S.d.N.; software, A.J.C.S.; validation, A.J.C.S. and J.A.S.d.N.; formal analysis, R.M.S.d.O., A.J.C.S. and J.A.S.d.N.; investigation, R.M.S.d.O., A.J.C.S. and J.A.S.d.N.; resources, R.M.S.d.O. and J.A.S.d.N.; data curation, A.J.C.S.; writing-original draft preparation, A.J.C.S.; writing-review and editing, R.M.S.d.O., A.J.C.S. and J.A.S.d.N.; visualization, A.J.C.S. and J.A.S.d.N.; supervision, R.M.S.d.O.; project administration, R.M.S.d.O.; funding acquisition, R.M.S.d.O. All authors have read and agreed to the published version of the manuscript.

Funding: This paper publication fee was funded by PROPESP/UFPA (Edital PAPQ).

Institutional Review Board Statement: Not applicable.

Informed Consent Statement: Not applicable.

Data Availability Statement: The authors reserve the right to not disclose the Eletronorte's private data sets of laboratorial PD measurements used in this study.

Acknowledgments: Authors would like to thank the Universidade Federal do Pará (UFPA) for financial support and Centrais Elétricas do Norte do Brasil (Eletronorte/Eletrobras) for granting permission to use their high voltage laboratory for developing the experimental part of this work.

Conflicts of Interest: The authors declare no conflicts of interest.

\section{Appendix A. Antenna Design}

In this work, we designed and used a patch antenna for covering the band between $0.33 \mathrm{GHz}$ and $2.02 \mathrm{GHz}$, since PD signals with frequencies over $1.5 \mathrm{GHz}$ were measured in [20]. Initially, a preliminary model of the a golden ratio patch antenna was developed, as is presented in Figure 11. The patch is represented using the blue color, the ground plane is depicted in green, and the dielectric substrate is outlined in gray. The substrate is manufactured using a Flame Retardant epoxy material (type 4), of which relative electric permittivity is 4.4 and its loss tangent is 0.02 . The antenna dimensions are $L=400 \mathrm{~mm}$ (length), $W=300 \mathrm{~mm}$ (width), and $H=1.5 \mathrm{~mm}$ (thickness).

The patch is formed by circles that are centered on the borders of smaller circles. The ratio of the radius of any circle to its immediately smaller circle is given by the golden ratio $r_{a}=1.618$. The geometric parameters of the proposed antenna are indicated in Figure 11. For the preliminary antenna model, the geometric parameters are $h_{p t}=57 \mathrm{~mm}, h_{s t}=10 \mathrm{~mm}$, $w_{s t}=6 \mathrm{~mm}, R_{1}=140 \mathrm{~mm}, R_{2}=R_{1} / r_{a}=86.52 \mathrm{~mm}$, and $R_{3}=R_{2} / r_{a}=53.47 \mathrm{~mm}$. The length of the microstrip feed line is $h_{p t}+1 \mathrm{~mm}$, and its width is $3 \mathrm{~mm}$. The parameter $L=40 \mathrm{~cm}$ was chosen in order to produce resonance at approximately $300 \mathrm{MHz}$. The three circles were used to achieve additional multiple resonance bands at higher frequencies. The ground plane also contributes with its high frequency resonance band. Consequently, a broadband antenna can be obtained by optimizing its geometric parameters, aiming to achieve proper merging of the resonance bands produced by each circular element, by the half perimeter of the patch, by the length of the feeding line, and by the ground plane dimensions.

Reflection coefficient $S_{11}$, obtained at the antenna terminals [41], is calculated in this work considering the reference impedance $Z_{0}=50 \Omega$. The reflection coefficients (in $\mathrm{dB}$ ) of the preliminary antenna model, calculated from COMSOL Multiphysics simulation, are presented in Figure A1 (curve $C_{1}$ ). As one can see in curve $C_{1}$, for the initial (preliminary) antenna model, $S_{11}$ undesirably crosses the reference level of $-10 \mathrm{~dB}$ around $0.8 \mathrm{GHz}$, near $1.3 \mathrm{GHz}$, and at $1.8 \mathrm{GHz}$. For this reason, optimization of the dimensional parameters of the initial antenna model were necessary to allow full coverage of the aimed PD frequency range (300-2000 MHz) [20]. 


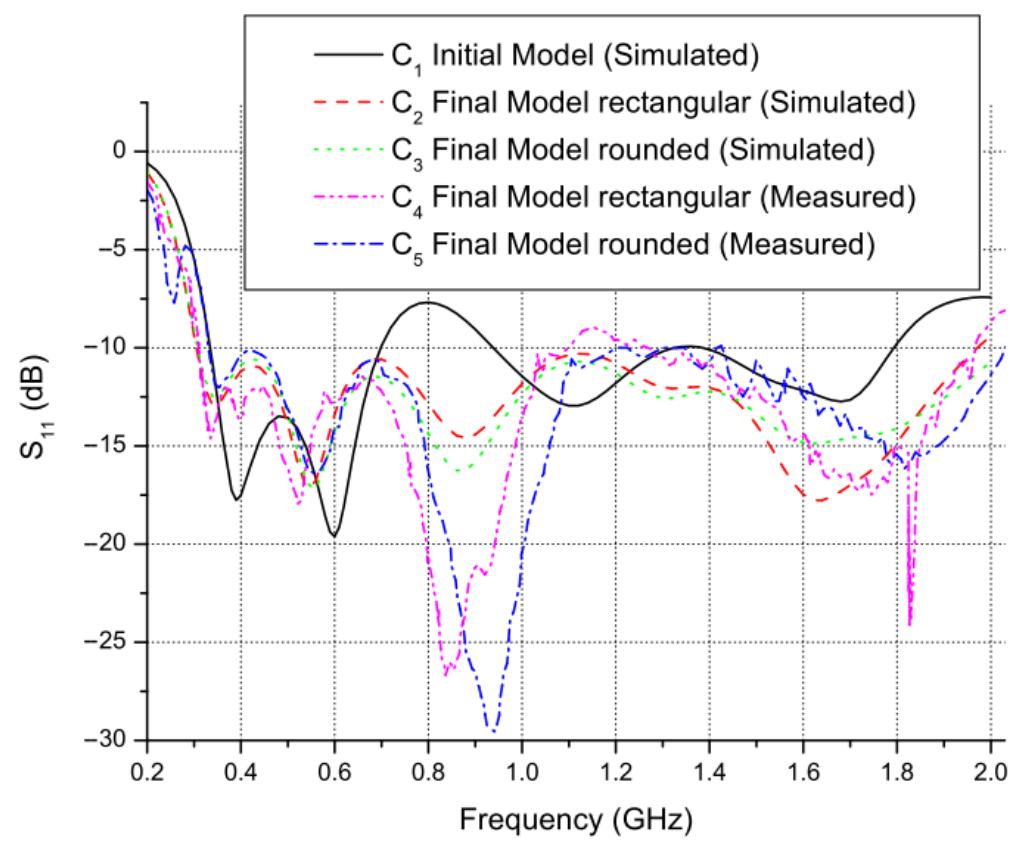

Figure A1. Simulated and measured reflection coefficients of the antennas.

Initial adjustments to the dimensional parameters were done by preserving other dimensions of the initial model; i.e., tuning was performed, at first, by varying a single parameter at a time. The results of these individual parametric sweeps of $h_{p t}, R_{1}, h_{s t}$, and $w_{s t}$ are presented in Figure A2. By setting $R_{1}$ to $135 \mathrm{~mm}$, reflection coefficient $S_{11}$ was greatly reduced around $0.8 \mathrm{GHz}$ with respect to the initial model of the antenna. However, with this adjustment on $R_{1}$, the parameter $S_{11}$ was increased to levels over $-10 \mathrm{~dB}$ in the band between $1.1 \mathrm{GHz}$ and $1.45 \mathrm{GHz}$. On the other hand, by setting $h_{s t}$ to $8 \mathrm{~mm}$, levels of $S_{11}$ in the band between $1.1 \mathrm{GHz}$ and $1.45 \mathrm{GHz}$ are well below $-10 \mathrm{~dB}$, as Figure A2 shows. Thus, an optimized antenna was obtained by performing a combination of parameters producing reflection coefficients under $-10 \mathrm{~dB}$ in frequency bands, in which the initial antenna model does not provide that characteristic and in regions of the spectrum in which a desired parameter modification produces undesired $S_{11}$ values in other frequency bands of interest. Thus, we have obtained the following optimized set of parameters: $h_{p t}=68 \mathrm{~mm}$, $h_{s t}=9 \mathrm{~mm}, w_{s t}=5 \mathrm{~mm}, R_{1}=135 \mathrm{~mm}, R_{2}=R_{1} / r_{a}=83.43 \mathrm{~mm}$, and $R_{3}=R_{2} / r_{a}=51.56 \mathrm{~mm}$.

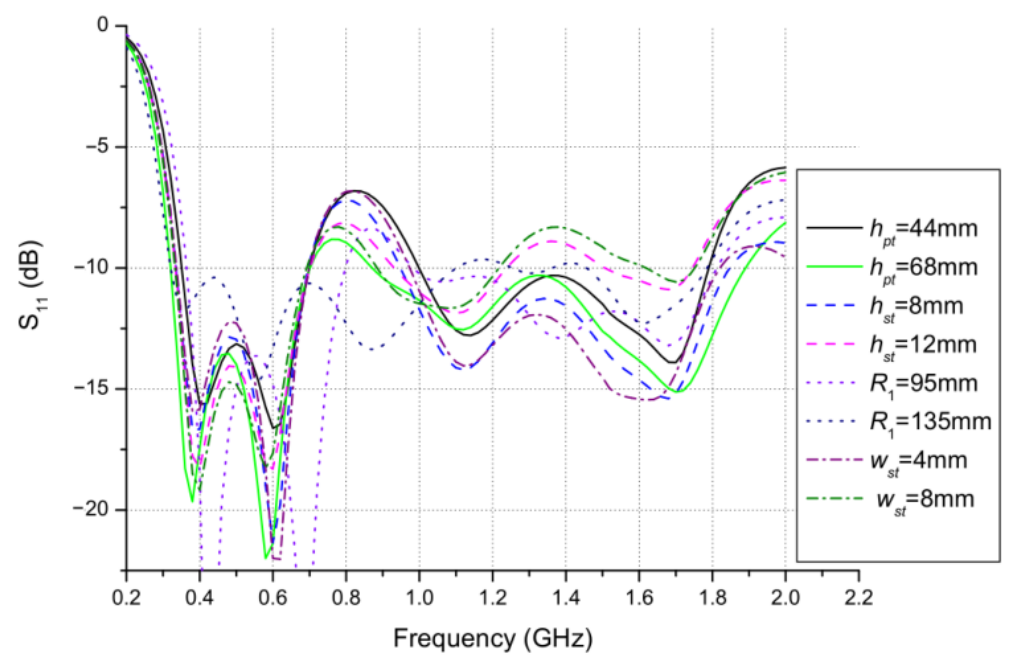

Figure A2. Simulated reflection coefficients regarding adjustments of dimensional parameters. 
Finally, the optimized antenna has been built, as Figure A3 illustrates. A vector network analyzer (VNA) has been used for measuring the parameter $S_{11}$ of the antenna, and the experimental results are compared with the numerical curve in Figure A1 (see curves $C_{2}$ and $C_{4}$ ). As one can observe, even with the obtained similarity between the measured and the simulated reflection coefficients, measured signal is above $-10 \mathrm{~dB}$ in the frequency band between approximately $1.1 \mathrm{GHz}$ and $1.25 \mathrm{GHz}$. As long as the ground plane width is $W=0.3 \mathrm{~m}$, this dimension is close to $\lambda / 2$ in the dielectric substrate at $1.25 \mathrm{GHz}$. As a consequence, the ground plane has an important influence on the antenna response around 1.25 GHz. Thus, in order to reduce the reflection coefficient in the frequency range of interest, the edges of the ground plane were modified to be rounded, as is shown in Figure A4. The comparison between the measured and simulated $S_{11}$ curves are presented in Figure $A 1$ (see curves $C_{3}$ and $C_{5}$ ). This final improvement introduced to the antenna geometry produces reflection coefficients measured under $-10 \mathrm{~dB}$ over the entire targeted PD frequency band, between $0.33 \mathrm{GHz}$ and $2.02 \mathrm{GHz}$. The obtained fractional bandwidth $(F B W)$ is $143.83 \%$.

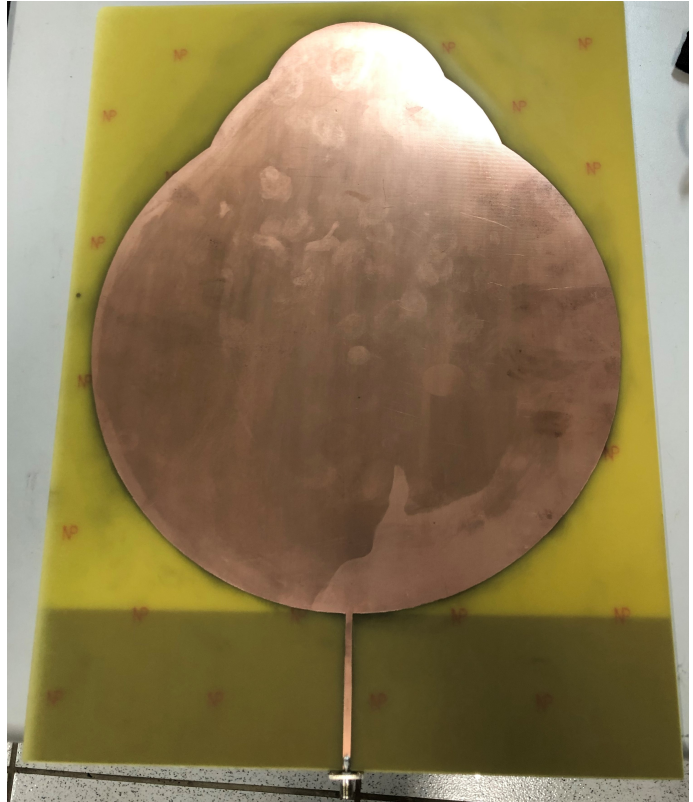

(a)

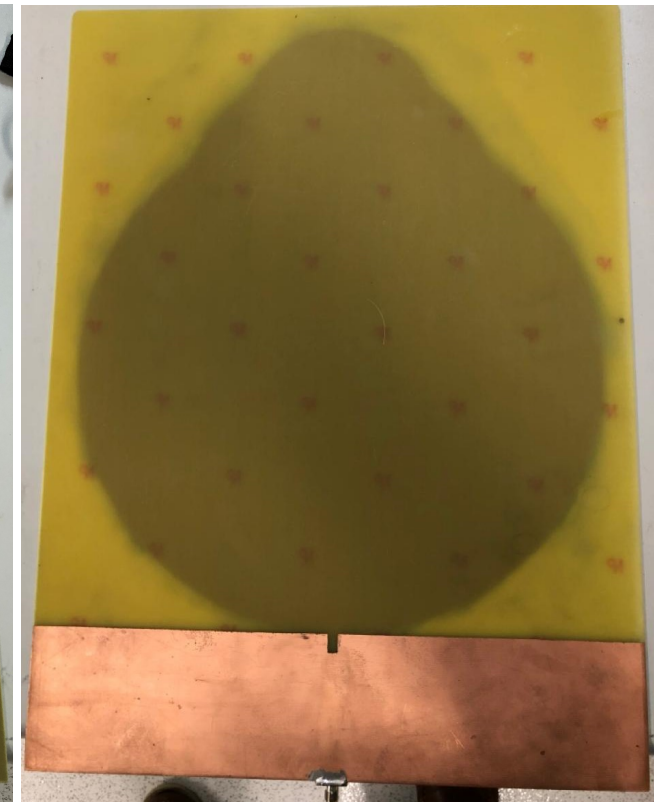

(b)

Figure A3. Optimized antenna designed with right angles ground plane: (a) front view and (b) back view.

Figure A5 shows real and imaginary parts of the final antenna's impedance $Z=R+j X$, where $R$ and $X$ are the resistance and the reactance of the antenna. One can confirm that the antenna satisfies desired impedance matching goals, since the imaginary part of the impedance is around $0 \Omega$, reaching maximum and minimum values of 25.16 and $-26.43 \Omega$, while the real part is around $Z_{0}=50 \Omega$, with maximum and minimum values of 73.33 and $27.75 \Omega$, respectively, in the frequency range of interest. This feature is confirmed by inspecting the curve $C_{5}$ Figure $A 1$, which is under $-10 \mathrm{~dB}$ in the aimed frequency range. 


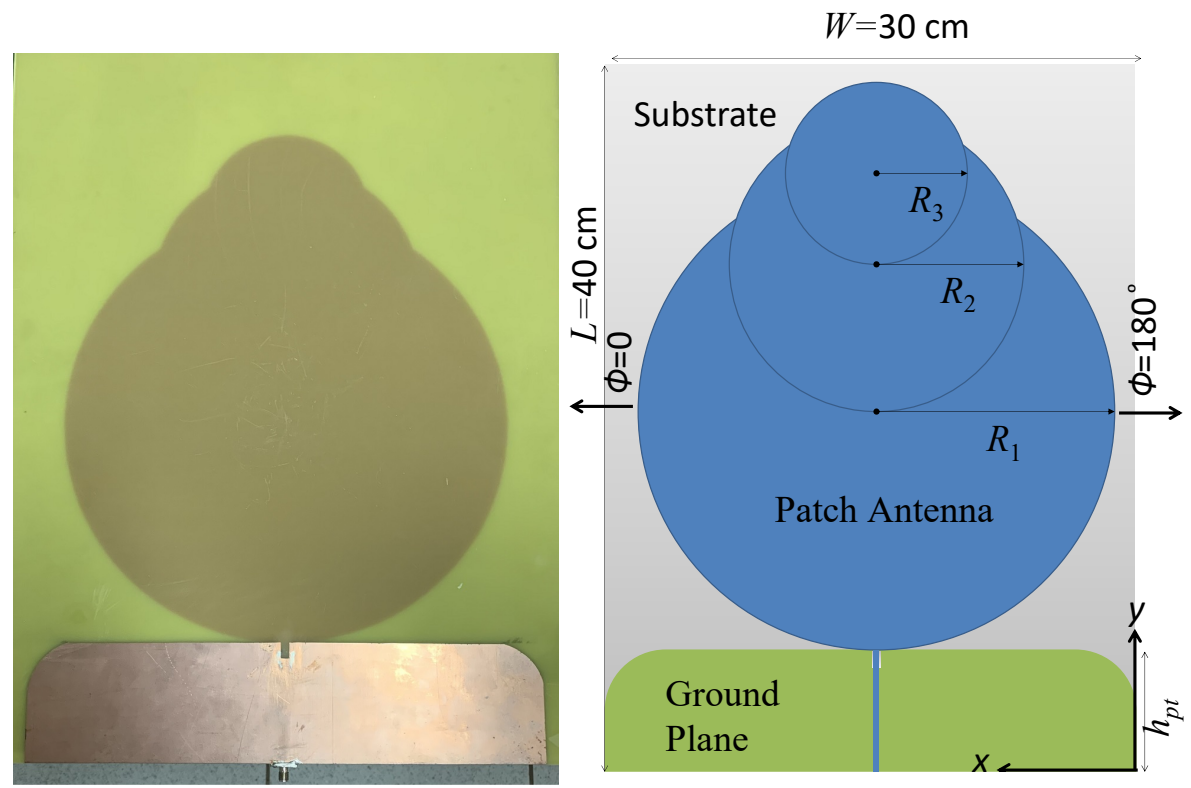

(a)

(b)

Figure A4. The final antenna, with rounded ground plane: (a) manufactured and (b) final geometric design.

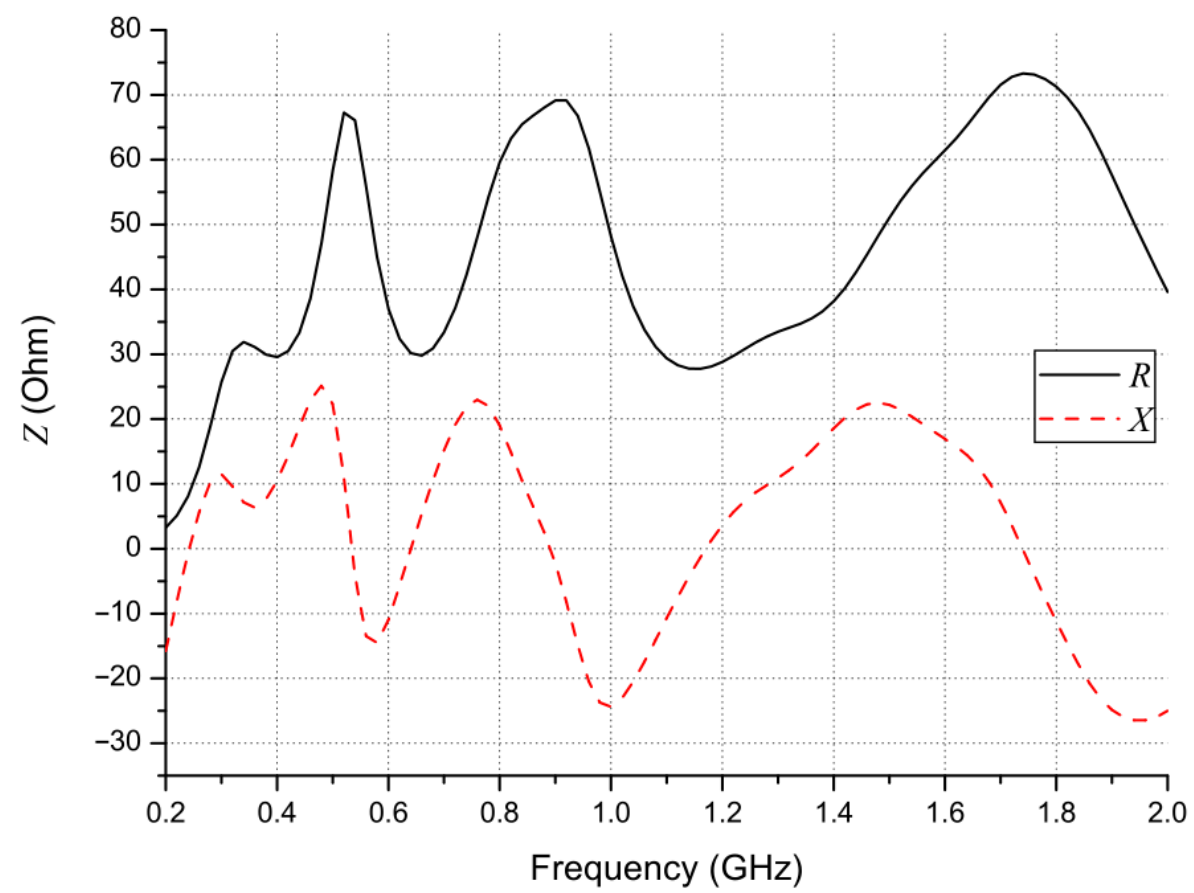

Figure A5. Simulated input impedance of the final antenna model.

Finally, for the sake of illustration, the three-dimensional radiation pattern of the antenna is shown in Figure A6, which was calculated at $450 \mathrm{MHz}$. Figure A7 shows maximum gain calculated as a function of frequency. 


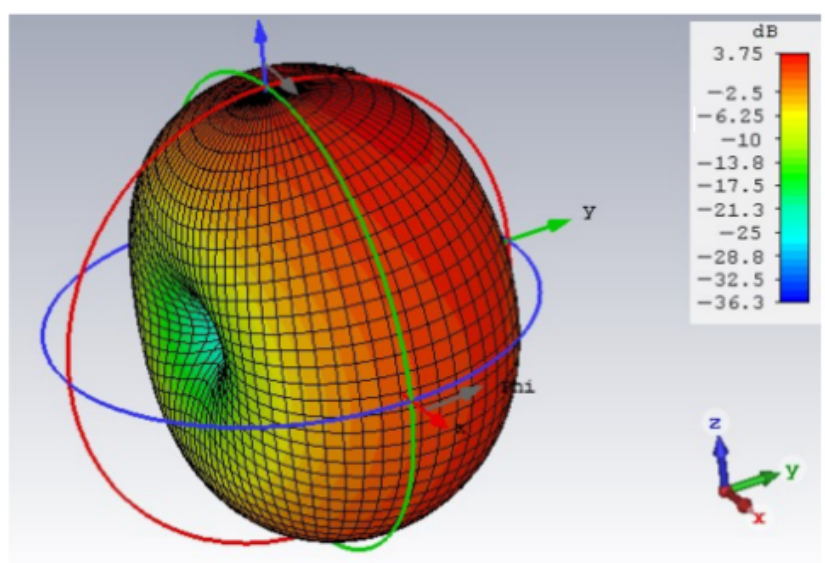

(a)

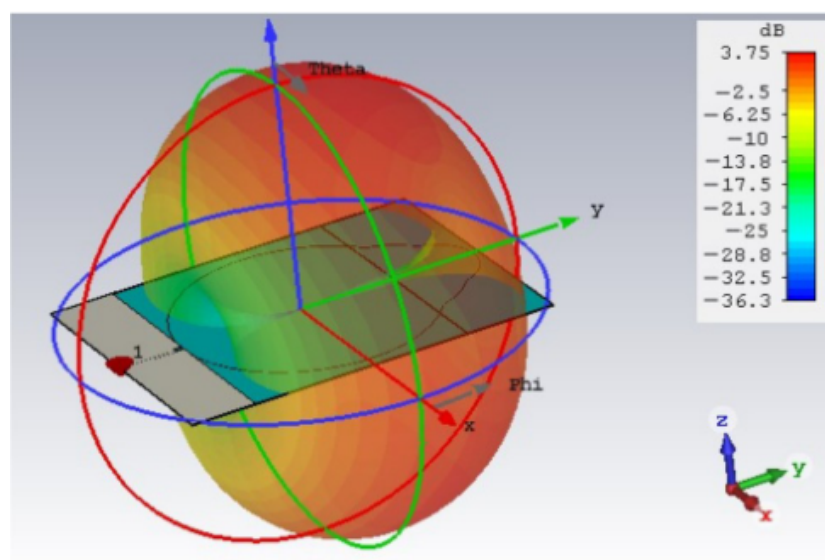

(b)

Figure A6. Simulated 3D radiation pattern of the antenna obtained at $450 \mathrm{MHz}$ : (a) standard diagram and $(\mathbf{b})$ radiation pattern and the patch antenna.

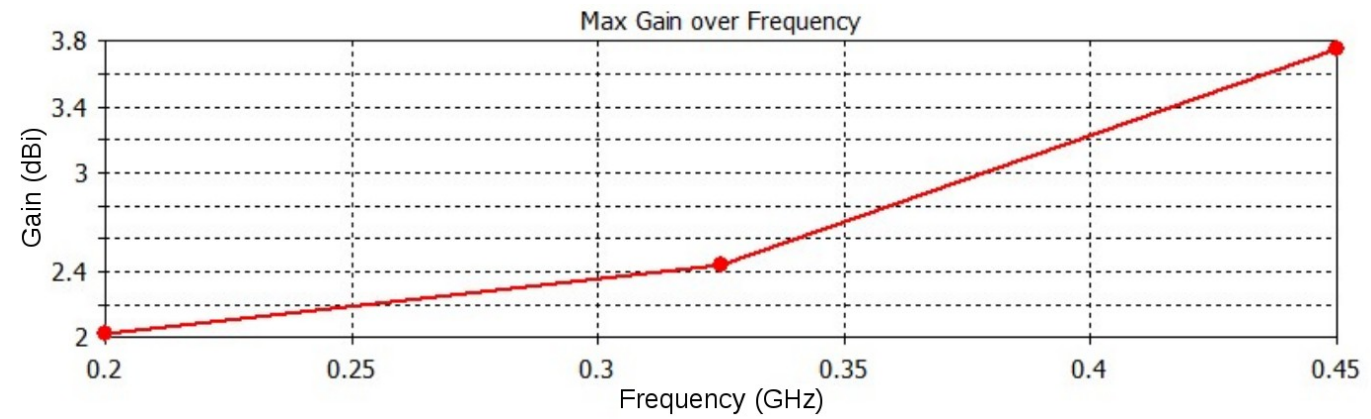

Figure A7. Maximum gain as a function of frequency. 


\section{References}

1. Stone, G. Condition Monitoring and Diagnostics of Motor and Stator Windings A Review. IEEE Trans. Dielectr. Electr. Insul. 2013, 20, 2073-2080. [CrossRef]

2. Stone, G.C.; Culbert, I.; Boulter, E.A.; Dhirani, H. Electrical Insulation for Rotating Machines, 2nd ed.; Wiley: Hoboken, NJ, USA, 2004.

3. Dehlinger, N.; Stone, G. Surface partial discharge in hydrogenerator stator windings: Causes, symptoms, and remedies. IEEE Electr. Insul. Mag. 2020, 36, 7-18. [CrossRef]

4. Cselko, R.; Tamus, Z.A.; Szabo, A.; Berta, I. Comparison of acoustic and electrical partial discharge measurements on cable terminations. In Proceedings of the 2010 IEEE International Symposium on Electrical Insulation, San Diego, CA, USA, 6-9 June 2010; pp. 1-5. [CrossRef]

5. Ilkhechi, H.D.; Samimi, M.H. Applications of the Acoustic Method in Partial Discharge Measurement: A Review. IEEE Trans. Dielectr. Electr. Insul. 2021, 28, 42-51. [CrossRef]

6. IEC/TS60034-27-2. Rotating Electrical Machines-Part 27-2: On-line Partial Discharge Measurements on the Stator Winding Insulation of Rotating Electrical Machines, 1st ed.; International Electrotechnical Commission: Geneva, Switzerland, 2012.

7. Araujo, R.C.F.; de Oliveira, R.M.S.; Brasil, F.S.; Barros, F.J.B. Novel Features and PRPD Image Denoising Method for Improved Single-Source Partial Discharges Classification in On-Line Hydro-Generators. Energies 2021, 14, 3267. [CrossRef]

8. Yadam, Y.R.; Ramanujam, S.; Arunachalam, K. An Ultrawideband Conical Monopole With Radome for Detection of Partial Discharges. IEEE Sens. J. 2021, 21, 18764-18772. [CrossRef]

9. Raymond, W.J.K.; Illias, H.A.; Bakar, A.H.A.; Mokhlis, H. Partial discharge classifications: Review of recent progress. Measurement 2015, 68, 164-181. [CrossRef]

10. Luo, Y.; Li, Z.; Wang, H. A Review of Online Partial Discharge Measurement of Large Generators. Energies 2017, 10, 1694. [CrossRef]

11. Lee, S.B.; Stone, G.C.; Antonino-Daviu, J.; Gyftakis, K.N.; Strangas, E.G.; Maussion, P.; Platero, C.A. Condition Monitoring of Industrial Electric Machines: State of the Art and Future Challenges. IEEE Ind. Electron. Mag. 2020, 14, 158-167. [CrossRef]

12. Contin, A.; Montanari, G.; Ferraro, C. PD source recognition by Weibull processing of pulse height distributions. IEEE Trans. Dielectr. Electr. Insul. 2000, 7, 48-58. [CrossRef]

13. Dey, D.; Chatterjee, B.; Chakravorti, S.; Munshi, S. Rough-granular approach for impulse fault classification of transformers using cross-wavelet transform. IEEE Trans. Dielectr. Electr. Insul. 2008, 15, 1297-1304. [CrossRef]

14. Peng, X.; Wen, J.; Li, Z.; Yang, G.; Zhou, C.; Reid, A.; Hepburn, D.M.; Judd, M.D.; Siew, W.H. Rough set theory applied to pattern recognition of Partial Discharge in noise affected cable data. IEEE Trans. Dielectr. Electr. Insul. 2017, 24, 147-156. [CrossRef]

15. Stone, G.; Culbert, I.; Bolter, E.; Dhirani, H. Electrical Insulation for Rotating Machines: Design, Evaluation, Aging, Testing, and Repair, 2nd ed.; IEEE Press: Piscataway, NJ, USA; Wiley: Hoboken, NJ, USA, 2014.

16. Dakin, T.W. The Relation of Capacitance Increase with High Voltages to Internal Electric Discharges and Discharging Void Volume. Trans. Am. Inst. Electr. Eng. Part III Power Appar. Syst. 1959, 78, 790-794. [CrossRef]

17. Fruth, B.; Niemeyer, L.; Hassig, M.; Fuhr, J.; Dunz, T. Phase Resolved Partial Discharge Measurements and Computer Aided Partial Discharge Analysis Performed on Different High Voltage Apparatus. In Proceedings of Sixth Int. Symp. on High Voltage Engineering, 6th ISH, New Orleans, LA, USA, 28 August-1 September 1989; pp. 15.03-15.06.

18. Oliveira, R.M.S.; Araujo, R.C.F.; Segundo, A.P.M.; Zampolo, R.F.; Fonseca, W.; Dmitriev, V.; Brasil, F.S. A System Based on Artificial Neural Networks for Automatic Classification of Hydro-generator Stator Windings Partial Discharges. J. Microwaves Optoelectron. Electromagn. Appl. 2017, 16, 628-645. [CrossRef]

19. Hudon, C.; Belec, M. Partial Discharges Signal Interpretation for Generator Diagnostics. IEEE Trans. Dielectr. Electr. Insul. 2005, 12, 297-319. [CrossRef]

20. Sako, H.; Mio, K.; Okada, S. Analysis of Phase Resolved Partial Discharge patterns with microstrip antenna. In Proceedings of the 2015 IEEE Electrical Insulation Conference (EIC), Seattle, WA, USA, 27 August 2015; pp. 346-357. [CrossRef]

21. Bartnikas, R. Corona Discharge Processes in Voids. In Engineering Dielectrics: Corona Measurement and Interpretation, 2nd ed.; Bartinikas, R., Macmahon, E.J., Eds.; ASTM: West Conshohocken, PA, USA, 1979; Chapter 2, Volume I.

22. Raizer, Y.P. Gas Discharge Physics; Springer-Verlag: Berlin/Heidelberg, Germany, 1991.

23. Meek, J.M.; Craggs, J.D. Electrical Breakdown of Gases; Clarendon Press: Oxford, UK, 1953; pp. 251-290.

24. Balanis, C.A. Advanced Engineering Eletromagnetics; John Wiley and Sons: New York, NY, USA, 1989.

25. Eichwald, O.; Ducasse, O.; Dubois, D.; Abahazem, A.; Merbahi, N.; Benhenni, M.; Yousfi, M. Experimental analysis and modelling of positive streamer in air: towards an estimation of $\mathrm{O}$ and $\mathrm{N}$ radical production. J. Phys. Appl. Phys. 2008. [CrossRef]

26. Sigmond, R.S. The residual streamer channel: return strokes and secondary streamers. J. Appl. Phys. 1984, 56, 1355-1370. [CrossRef]

27. Huiskamp, T.; Sengers, W.; Beckers, F.J.C.M.; Nijdam, S.; Ebert, U.; van Heesch, E.J.M.; Pemen, A.J.M. Spatiotemporally resolved imaging of streamer discharges in air generated in a wire-cylinder reactor with (sub)nanosecond voltage pulses. Plasma Sources Sci. Technol. 2017, 26, 075009. [CrossRef]

28. Hudson, G.G.; Loeb, L.B. Streamer Mechanism and Main Stroke in the Filamentary Spark Breakdown in Air as Revealed by Photomultipliers and Fast Oscilloscopic Techniques. Phys. Rev. 1961, 123, 1. [CrossRef] 
29. IEC60270. High Voltage Test Techniques_Partial Discharge Measurements, 3rd ed.; International Electrotechnical CommissionBritish Standard: London, UK, 2001.

30. Schneider, K.; Farge, M. Wavelets: Mathematical Theory. In Encyclopedia of Mathematical Physics; Francoise, J.P.; Naber, G.L.; Tsun, T.S., Eds.; Academic Press: Oxford, UK, 2006; pp. 426-438. [CrossRef]

31. Oliveira, R.M.S.; Modesto, J.F.M.; Dmitriev, V.; Brasil, F.S.; Vilhena, P.R.M. Spectral Method for Localization of Multiple Partial Discharges in Dielectric Insulation of Hydro-Generator Coils: Simulation and Experimental Results. J. Microwaves Optoelectron. Electromagn. Appl. 2016, 15, 170-190. [CrossRef]

32. Sadiku, M. Elements of Electromagnetics, 3rd ed.; Oxford University Press: Oxford, UK, 2000.

33. Yang, F.; Peng, C.; Yang, Q.; Luo, H.; Ullah, I.; Yang, Y. An UWB printed antenna for partial discharge UHF detection in high voltage switchgears. Prog. Electromagn. Res. C 2016, 69, 105-114. [CrossRef]

34. Xavier, G.; Costa, E.; Serres, A.; Nobrega, L.; Oliveira, A.; Sousa, H. Design and Application of a Circular Printed Monopole Antenna in Partial Discharge Detection. IEEE Sens. J. 2019, 19, 3718-3725. [CrossRef]

35. Zhang, Y.; Lazaridis, P.; Abd-Alhameed, R.; Glover, I. A compact wideband printed antenna for free-space radiometricdetection of partial discharge. Turk. J. Electr. Eng. Comput. Sci. 2017, 25, 1291-1299. [CrossRef]

36. Dunlap, R.A. The Golden Ratio and Fibonacci Numbers; World Scientific Publishing Company: Toh Tuck Link, Singapore, 1998.

37. Gupta, S.; Arora, T.; Singh, D.; Kumar Singh, K. Nature Inspired Golden Spiral Super-Ultra Wideband Microstrip Antenna. In Proceedings of the 2018 Asia-Pacific Microwave Conference (APMC), Kyoto, Japan, 6 November 2018; pp. 1603-1605. [CrossRef]

38. Meena, A.; Garhwal, A.; Ray, K. Nature Inspired Fibonacci Sequence Microstrip Patch Antenna For Energy Harvesting Applications. Int. J. Recent Technol. Eng. (IJRTE) 2019, 8, 6154-6160.

39. Tanabe, M.; Nakano, H. Low-Profile Wideband Spiral Antenna With a Circular HIS Reflector Composed of Homogenous Fan-Shaped Patch Elements. IEEE Trans. Antennas Propag. 2020, 68, 7219-7222. [CrossRef]

40. Soboll, P.; Wienstroer, V.; Kronberger, R. Smooth Moves in Power Transition: New Yagi-Uda Antenna Design for Wireless Energy. IEEE Microw. Mag. 2016, 17, 75-80. [CrossRef]

41. Balanis, C.A. Fundamental Parameters of Antennas. In Antenna Theory: Analysis and Design;Wiley-Interscience: Hoboken, NJ, USA, 2005; Chapter 2, pp. 27-132. 